\title{
Biplanes with flag-transitive automorphism groups of almost simple type, with classical socle
}

\author{
Eugenia O'Reilly-Regueiro
}

Received: 31 October 2005 / Accepted: 9 March 2007 /

Published online: 21 April 2007

(C) Springer Science+Business Media, LLC 2007

\begin{abstract}
In this paper we prove that if a biplane $D$ admits a flag-transitive automorphism group $G$ of almost simple type with classical socle, then $D$ is either the unique $(11,5,2)$ or the unique $(7,4,2)$ biplane, and $G \leq P S L_{2}(11)$ or $P S L_{2}(7)$, respectively. Here if $X$ is the socle of $G$ (that is, the product of all its minimal normal subgroups), then $X \unlhd G \leq$ Aut $G$ and $X$ is a simple classical group.
\end{abstract}

Keywords Automorphism group $\cdot$ Biplanes $\cdot$ Flag-transitive

\section{Introduction}

A biplane is a $(v, k, 2)$-symmetric design, that is, an incidence structure of $v$ points and $v$ blocks such that every point is incident with exactly $k$ blocks, and every pair of blocks is incident with exactly two points. Points and blocks are interchangeable in the previous definition, due to their dual role. A nontrivial biplane is one in which $2<k<v-1$. A flag of a biplane $D$ is an ordered pair $(p, B)$ where $p$ is a point of $D, B$ is a block of $D$, and they are incident. Hence if $G$ is an automorphism group of $D$, then $G$ is flag-transitive if it acts transitively on the flags of $D$.

The only values of $k$ for which examples of biplanes are known are $k=3,4,5,6$, 9,11 , and 13 [7, pp. 76]. Due to arithmetical restrictions on the parameters, there are no examples with $k=7,8,10$, or 12 .

For $k=3,4$, and 5 the biplanes are unique up to isomorphism [6], for $k=6$ there are exactly three non-isomorphic biplanes [13], for $k=9$ there are exactly four nonisomorphic biplanes [26], for $k=11$ there are five known biplanes [3, 10, 11], and for $k=13$ there are two known biplanes [1], in this case, it is a biplane and its dual.

E. O’Reilly-Regueiro ( $₫)$

Instituto de Matemáticas, Universidad Nacional Autónoma de México, Mexico, DF 04510,

Mexico

e-mail: eugenia@matem.unam.mx 
In [24] it is shown that if a biplane admits an imprimitive, flag-transitive automorphism group, then it has parameters $(16,6,2)$. There are three non-isomorphic biplanes with these parameters [4], two of which admit flag-transitive automorphism groups which are imprimitive on points, (namely $2^{4} S_{4}$ and $\left(\mathbb{Z}_{2} \times \mathbb{Z}_{8}\right) S_{4}$ [24]). Therefore, if any other biplane admits a flag-transitive automorphism group $G$, then $G$ must be primitive. The O'Nan-Scott Theorem classifies primitive groups into five types [22]. It is shown in [24] that if a biplane admits a flag-transitive, primitive, automorphism group, it can only be of affine or almost simple type. The affine case was treated in [24]. The almost simple case when the socle of $G$ is an alternating or a sporadic group was treated in [25], in which it is shown that no such biplane exists. Here we treat the almost simple case when the socle $X$ of $G$ is a classical group. We now state the main result of this paper:

Theorem 1 (Main Theorem) If $D$ is a nontrivial biplane with a primitive, flagtransitive automorphism group $G$ of almost simple type with classical socle $X$, then $D$ has parameters either $(7,4,2)$, or $(11,5,2)$, and is unique up to isomorphism.

This, together with [24, Theorem 3] and [25, Theorem 1] yield the following:

Corollary 1 If $D$ is a nontrivial biplane with a flag-transitive automorphism group $G$, then one of the following holds:

(1) D has parameters $(7,4,2)$,

(2) D has parameters $(11,5,2)$,

(3) D has parameters $(16,6,2)$,

(4) $G \leq A \Gamma L_{1}(q)$, for some odd prime power $q$, or

(5) $G$ is of almost simple type, and the socle $X$ of $G$ is an exceptional group of Lie type.

For the purpose of proving our Main Theorem, we will consider $D$ to be a nontrivial biplane, with a primitive, flag-transitive, almost simple automorphism group $G$, with simple socle $X$, such that $X=X_{d}(q)$ is a simple classical group, with a natural projective action on a vector space $V$ of dimension $d$ over the field $\mathbb{F}_{q}$, where $q=p^{e}$, ( $p$ prime).

For this we will proceed as in [27], in which the case for finite linear spaces with almost simple flag-transitive automorphism groups of Lie type is treated.

\section{Preliminary results}

In this section we state some preliminary results we will use throughout this paper.

Lemma 2 If $D$ is a $(v, k, 2)$-biplane, then $8 v-7$ is a square.

Proof The result follows from [24, Lemma 3].

Corollary 3 If $D$ is a flag-transitive $(v, k, 2)$-biplane, then $2 v<k^{2}$, and hence $2|G|<\left|G_{x}\right|^{3}$. 
Proof The equality $k(k-1)=2(v-1)$, implies $k^{2}=2 v-2+k$, so clearly $2 v<k^{2}$. The result follows from $v=\left|G: G_{x}\right|$ and $k \leq\left|G_{x}\right|$.

From [9] we get the following two lemmas:

Lemma 4 If $D$ is a biplane with a flag-transitive automorphism group $G$, then $k$ divides $2 d_{i}$ for every subdegree $d_{i}$ of $G$.

Lemma 5 If $G$ is a flag-transitive automorphism group of a biplane $D$, then $k$ divides $2 \cdot \operatorname{gcd}\left(v-1,\left|G_{x}\right|\right)$.

Lemma 6 (Tits Lemma [28, 1.6]) If X is a simple group of Lie type in characteristic $p$, then any proper subgroup of index prime to $p$ is contained in a parabolic subgroup of $X$.

Lemma 7 If $X$ is a simple group of Lie type in characteristic $2,\left(X \nsubseteq A_{5}\right.$ or $\left.A_{6}\right)$, then any proper subgroup $H$ such that $[X: H]_{2} \leq 2$ is contained in a parabolic subgroup of $X$.

Proof First assume $X=C l_{n}(q)$ is classical ( $q$ a power of 2), and take $H$ maximal in $X$. By Aschbacher's Theorem [2], $H$ is contained in a member of the collection $\mathcal{C}$ of subgroups of $\Gamma L_{n}(q)$, or in $\mathcal{S}$, that is, $H^{(\infty)}$ is quasisimple, absolutely irreducible, and not realisable over any proper subfield of $\mathbb{F}(q)$.

We check for every family $\mathcal{C}_{i}$ that if $H$ is contained in $C_{i}$, then $2|H|_{2}<|X|_{2}$, except when $H$ is parabolic.

Now we take $H \in \mathcal{S}$. Then by [18, Theorem 4.2], $|H|<q^{2 n+4}$, or $H$ and $X$ are as in [18, Table 4]. If $|X|_{2} \leq 2|H|_{2} \leq q^{2 n+4}$, then either $X=L_{n}^{\epsilon}(q)$ and $n \leq 6$, or $X=S p_{n}(q)$ or $P \Omega_{n}^{\epsilon}(q)$ and $n \leq 10$. We check the list of maximal subgroups of $X$ for $n \leq 10$ in [15, Chapter 5], and we see that no group $H$ satisfies $2|H|_{2} \leq|X|_{2}$. We then check the list of groups in [18, Table 4], and again, none of them satisfy this bound.

Finally, assume $X$ to be an exceptional group of Lie type in characteristic 2 . By [20], if $2|H| \geq|X|_{2}$, then $H$ is either contained in a parabolic subgroup, or $H$ and $X$ are as in [20, Table 1]. Again, we check all the groups in [20, Table 1], and in all cases $2|H|_{2}<|X|_{2}$.

As a consequence, we have a strengthening of Corollary 3:

Corollary 8 Suppose $D$ is a biplane with a primitive, flag-transitive almost simple automorphism group $G$ with simple socle $X$ of Lie type in characteristic $p$, and the stabiliser $G_{x}$ is not a parabolic subgroup of $G$. If $p$ is odd then $p$ does not divide $k$; and if $p=2$ then 4 does not divide $k$. Hence $|G|<2\left|G_{x}\right|\left|G_{x}\right|_{p^{\prime}}^{2}$.

Proof We know from Corollary 3 that $|G|<\left|G_{x}\right|^{3}$. Now, by Lemma 6, $p$ divides $v=\left[G: G_{x}\right]$. Since $k$ divides $2(v-1)$, if $p$ is odd then $(k, p)=1$, and if $p=2$ then $(k, p) \leq 2$. Hence $k$ divides $2\left|G_{x}\right|_{p^{\prime}}$, and since $2 v<k^{2}$, we have $|G|<2\left|G_{x}\right|\left|G_{x}\right|_{p^{\prime}}^{2}$. 
From the previous results we have the following lemma, which will be quite useful throughout this chapter:

Lemma 9 Suppose p divides $v$, and $G_{x}$ contains a normal subgroup $H$ of Lie type in characteristic $p$ which is quasisimple and $p \nmid|Z(H)|$; then $k$ is divisible by $[H: P]$, for some parabolic subgroup $P$ of $H$.

Proof The assumption that $p$ divides $v$ and the fact that $k$ divides $2(v-1)$ imply $(k, p) \leq(2, p)$. Also, we know $k=\left[G_{x}: G_{x, B}\right]$ (where $B$ is a block incident with $x)$, so $\left[H: H_{B}\right]$ divides $k$, and therefore $\left(\left[H: H_{B}\right], p\right) \leq(2, p)$. By Lemmas 6 and 7 we conclude that $H_{B}$ is contained in a parabolic subgroup $P$ of $H$, and $P$ maximal in $H$ implies that $H_{B}$ is contained in $P$, so $k$ is divisible by $[H: P]$.

Lemma 10 ([21, 3.9]) If $X$ is a group of Lie type in characteristic $p$, acting on the set of cosets of a maximal parabolic subgroup, and $X$ is not $P S L_{d}(q), P \Omega_{2 m}^{+}(q)$ (with $m$ odd), nor $E_{6}(q)$, then there is a unique subdegree which is a power of $p$.

\section{$3 X$ is a linear group}

In this case we consider the socle of $G$ to be $P S L_{n}(q)$, and $\beta=\left\{v_{1}, v_{2}, \ldots, v_{n}\right\}$ a basis for the natural $n$-dimensional vector space $V$ for $X$.

Lemma 11 If the group $X$ is $P S L_{2}(q)$, then it is one of the following:

(1) $\mathrm{PSL}_{2}$ (7) acting on the $(7,4,2)$ biplane with point stabiliser $S_{4}$, or

(2) $P S L_{2}(11)$ acting on a $(11,5,2)$ biplane with point stabiliser $A_{5}$.

Proof Suppose $X \cong P S L_{2}(q),\left(q=p^{m}\right)$ is the socle of a flag-transitive automorphism group of a biplane $D$, so $G \leq P \Gamma L_{2}(q)$. As $G$ is primitive, $G_{x}$ is a maximal subgroup of $G$, and hence $X_{x}$ is isomorphic to one of the following [12]: (Note that $\left|G_{x}\right|$ divides $\left.(2, q-1) m\left|X_{x}\right|\right)$ :

(1) A solvable group of index $q+1$.

(2) $D_{(2, q)(q-1)}$.

(3) $D_{(2, q)(q+1)}$.

(4) $L_{2}\left(q_{0}\right)$ if $(r>2)$, or $P G L_{2}\left(q_{0}\right)$ if $(r=2)$, where $q=q_{0}^{r}, r$ prime.

(5) $S_{4}$ if $q=p \equiv \pm 1(\bmod 8)$.

(6) $A_{4}$ if $q=p \equiv 3,5,13,27,37(\bmod 40)$.

(7) $A_{5}$ if $q \equiv \pm 1(\bmod 10)$.

(1) Here $v=q+1$, so $k(k-1)=2(v-1)=2 q$, hence $q=3$, but $P S L_{2}(3)$ is not simple.

(2) and (3) The degrees in these cases are a triangular number, but the number of points on a biplane is always one more than a triangular number.

(4) First assume $r>2$. Clearly, $q_{0}$ divides $v=q_{0}^{r-1}\left(\frac{q_{0}^{2 r}-1}{q_{0}^{2}-1}\right)$, so $k$ divides $2\left(v-1, m q_{0}\left(q_{0}^{2}-1\right)\right)$, hence $k=\frac{2 m\left(q_{0}^{2}-1\right)}{n}$ for some $n$. Say $q_{0}=p^{b}$, so $m=b r$ and (except for $p=2$ and $2 \leq b \leq 4)$, we have $b<\sqrt{q_{0}}$, (since $b^{2}<p^{b}=q_{0}$ ). 
Now, $k^{2}>2 v$ implies

$$
\frac{4 m^{2}\left(q_{0}^{2}-1\right)^{2}}{n^{2}}>2 q_{0}^{r-1}\left(\frac{q_{0}^{2 r}-1}{q_{0}^{2}-1}\right),
$$

so

$$
n^{2}<\frac{2 m^{2}\left(q_{0}^{2}-1\right)^{3}}{\left(q_{0}^{2 r}-1\right) q_{0}^{r-1}} .
$$

First consider $r>3$, so $(r \geq 5)$. Here $q_{0}^{r}>b^{2} r^{2}=m^{2}$. On the other hand, $2 m^{2}>$ $\frac{q_{0}^{r-1}\left(q_{0}^{2 r}-1\right)}{\left(q_{0}^{2}-1\right)^{3}}$, therefore

$$
2 q_{0}^{r}<\frac{q_{0}^{r-1}\left(q_{0}^{2 r}-1\right)}{\left(q_{0}^{2}-1\right)^{3}}
$$

which is a contradiction.

Next consider $r=3$. From $k^{2}>2 v$, we obtain $18 b^{2}\left(q_{0}^{2}-1\right)^{3}>n^{2} q_{0}^{2}\left(q_{0}^{6}-1\right)$, this together with $b^{2}<q_{0}$, imply $n^{2}\left(q_{0}^{6}-1\right)<18 q_{0}^{5}$, therefore $q_{0} \leq 17$. We check for all possible values of $q_{0}$ that $8 v-7$ is not a square, contradicting Lemma 2.

Now assume $r=2$. Then $v=\frac{q_{0}\left(q_{0}^{2}+1\right)}{(2, q-1)}$. As $q=q_{0}^{2} \neq 2$, we have $m^{2}<q$, so $4 b^{2}<$ $q_{0}^{2}$, which implies $q_{0} \neq 2$.

First consider $q$ even. From $2(v-1)=k(k-1)$, we have $2\left(q_{0}^{3}+q_{0}-1\right)=$ $\frac{2 m\left(q_{0}^{2}-1\right)}{n}\left(\frac{2 m\left(q_{0}^{2}-1\right)}{n}-1\right)$, however $\operatorname{gcd}\left(q_{0}^{3}+q_{0}-1, q_{0}^{2}-1\right)$ divides 3 , which implies $k=\frac{6 m}{t}$, with $t=1,3$.

If $t=3$ then $q_{0}^{3}+q_{0}-1=2 m^{2}-m=m(2 m-1)<2 m^{2}$, but $m<q_{0}$, so this is a contradiction.

If $t=1$ then $q_{0}^{3}+q_{0}-1=18 m^{2}-6 m$, which implies $q_{0}<18$, that is $q_{0}=4,8$, or 16 . However $m=2 b$ implies $k=12 b$, so $v-1$ is divisible by 6 , but this is not the case for any of these values of $q_{0}$.

Now consider $q$ odd. The equality $2(v-1)=k(k-1)$ yields $q_{0}^{3}+q_{0}-2=$ $\frac{4 m^{2}}{n^{2}}\left(q_{0}^{2}-1\right)^{2}-\frac{2 m}{n}\left(q_{0}^{2}-1\right)$, and the inequality $k^{2}>2 v$ implies $\frac{4 m^{2}}{n^{2}}\left(q_{0}^{2}-1\right)^{2}>$ $q_{0}\left(q_{0}^{2}+1\right)$. In this case $m=2 b$, so $k=\frac{4 b\left(p^{2 b}-1\right)}{n}$, and $v=\frac{p^{3 b}+p^{b}}{2}>\frac{b^{6}+b^{2}}{2}$, hence we have the following inequalities:

$$
b^{6}+b^{2}<p^{3 b}+p^{b}<\frac{4 b\left(p^{2 b}-1\right)}{n}<\frac{4 b \cdot p^{2 b}}{n} .
$$

This implies $\frac{n\left(p^{3 b}+p^{b}\right)}{p^{2 b}}<4 b$, so $n\left(p^{b}+p^{\frac{b}{2}}\right)<4 b<4 p^{\frac{b}{2}}$, therefore $n\left(p^{\frac{b}{2}}+1\right)<4$ which implies $n=1=b$, and $p=3,5$, or 7 , but in all these cases $k>v$, which is a contradiction.

(5) In this case $q=p \equiv \pm 1(\bmod 8)$, and $m=1$, so $G_{0} \cong S_{4}$. We have $q$ odd, $v=\frac{q\left(q^{2}-1\right)}{48}$, and $k$ divides $2\left(\frac{q\left(q^{2}-1\right)-48}{48}, 24\right)$, so $k \mid 48$. Now $k^{2}>2 v$ implies $q \leq 37$, hence $q=7,17,23$, or 31 . The only one of these values for which $8 v-7$ is a square (Lemma 2) is $q=7$, so $v=7$ and $k=4$, that is, we have the $(7,4,2)$ biplane and $G=X \cong P S L_{2}(7)$. 
(6) Here $q=p \equiv 3,5,13,27$, or $37(\bmod 40)$, so $m=1$ and $G_{x} \cong A_{4}$. Here $v=\frac{q\left(q^{2}-1\right)}{24}$, and so $k$ divides $2\left(\frac{q\left(q^{2}-1\right)-24}{24}, 12\right)$, so $k \mid 24$. As $2 v<k^{2}$, we have $q=3,5$, or 13 . For $q=3$ we have $v=1$, which is a contradiction. For $q=5$ we have $v=5$, but there is no such biplane. Finally, $q=13$ implies $v=91$, but then $8 v-7$ is not a square, contradicting Lemma 2 .

(7) Here $q=p$ or $p^{2} \equiv \pm 1(\bmod 10)$, and $v=\frac{q\left(q^{2}-1\right)}{120}$, so $k$ divides $120 m$, with $m=1$ or 2 . The inequality $2 v<k^{2}$ implies $q^{3}-q<60 k^{2}<60(120)^{2} m^{2}$, so $q=9$, $11,19,29,31,41,49,59,61,71,79,81,89$, or 121 . Of these, the only value for which $8 v-7$ is a square is $q=11$. In this case, $v=11$ and $k=5$, that is, we have a $(11,5,2)$ biplane, with $G=X \cong P S L_{2}(11)$, and $G_{x} \cong A_{5}$.

This completes the proof of Lemma 11.

Lemma 12 The group $X$ is not $P S L_{n}(q)$, with $n>2$, and $(n, q) \neq(3,2)$.

Proof Suppose $X \cong P S L_{n}(q)$, with $n>2$ and $(n, q) \neq(3,2)$ (since $P S L_{3}(2) \cong$ $\left.P S L_{2}(7)\right)$. We have $q=p^{m}$, and take $\left\{v_{1}, \ldots, v_{n}\right\}$ to be a basis for the natural $n$-dimensional vector space $V$ for $X$. Since $G_{x}$ is maximal in $G$, then by Aschbacher's Theorem [2], the stabiliser $G_{x}$ lies in one of the families $\mathcal{C}_{i}$ of subgroups of $\Gamma L_{n}(q)$, or in the set $\mathcal{S}$ of almost simple subgroups not contained in any of these families. We will analyse each of these cases separately. In describing the Aschbacher subgroups, we denote by ${ }^{\wedge} H$ the pre-image of the group $H$ in the corresponding linear group.

$\mathcal{C}_{1}$ ) Here $G_{x}$ is reducible. That is, $G_{x} \cong P_{i}$ stabilises a subspace of $V$ of dimension $i$.

Suppose $G_{x} \cong P_{1}$. Then $G$ is 2 -transitive, and this case has already been done by Kantor [14].

Now suppose $G_{x} \cong P_{i}(1<i<n)$ fixes $W$, an $i$-subspace of $V$. We will assume $i \leq \frac{n}{2}$ since our arguments are arithmetic, and for $i$ and $n-i$ we have the same calculations. Considering the $G_{x}$-orbits of the $i$-spaces intersecting $W$ in $i$-1-dimensional spaces, we see $k$ divides

$$
\frac{2 q\left(q^{i}-1\right)\left(q^{n-i}-1\right)}{(q-1)^{2}} .
$$

Also,

$$
v=\frac{\left(q^{n}-1\right) \ldots\left(q^{n-i+1}-1\right)}{\left(q^{i}-1\right) \ldots(q-1)}>q^{i(n-i)},
$$

but $k^{2}>2 v$, so either $i=3$ and $n<10$, or $i=2$.

First assume $i=3$ and $q=2$.

If $n=9$ then $k=2^{2} \cdot 3^{2} \cdot 7^{2}$, but the equation $k(k-1)=2(v-1)$ does not hold.

If $n=8$ then $k=4 \cdot 7 \cdot 31$ but again the equation $k(k-1)=2(v-1)$ does not hold.

For $n=7 k=420$ or 210 , but again, $k$ does not divide $2(v-1)$.

Finally, if $n=6$ then $k=196$ or 98 , but neither is a divisor of $2(v-1)$.

Now assume $i=3$ and $q>2$. Then $n=6$ or 7 . 
If $n=7$ then $k$ divides

$$
2\left(\frac{q\left(q^{3}-1\right)\left(q^{4}-1\right)}{(q-1)^{2}}, \frac{\left(q^{7}-1\right)\left(q^{6}-1\right)\left(q^{5}-1\right)}{\left(q^{3}-1\right)\left(q^{2}-1\right)(q-1)}-1\right),
$$

but then $k^{2}<v$, which is a contradiction.

If $n=6$ then $k$ divides

$$
2\left(\frac{q\left(q^{3}-1\right)^{2}}{(q-1)^{2}}, \frac{\left(q^{6}-1\right)\left(q^{5}-1\right)\left(q^{4}-1\right)}{\left(q^{3}-1\right)\left(q^{2}-1\right)(q-1)}-1\right),
$$

But again $k^{2}<2 v$.

Hence $i=2$. Here $v=\frac{\left(q^{n}-1\right)\left(q^{n-1}-1\right)}{\left(q^{2}-1\right)(q-1)}$, and $G$ has suborbits with sizes:

$\mid\{2$-subspaces $H: \operatorname{dim}(H \cap W)=1\} \mid=\frac{q(q+1)\left(q^{n-2}-1\right)}{q-1}$ and

$\mid\{2$-subspaces $H: H \cap W=\overline{0}\} \mid=\frac{q^{4}\left(q^{n-2}-1\right)\left(q^{n-3}-1\right)}{\left(q^{2}-1\right)(q-1)}$.

If $n$ is even then $k$ divides $\frac{q\left(q^{n-2}-1\right)}{\left(q^{2}-1\right)}$, since $q+1$ is prime to $\frac{\left(q^{n-3}-1\right)}{q-1}$, this implies $k^{2}<v$, which is a contradiction.

Hence $n$ is odd, and $k$ divides $\frac{2 q\left(q^{n-2}-1\right)}{q-1}\left(q+1, \frac{n-3}{2}\right)$.

First assume $n=5$. Then $v=\left(q^{2}+1\right)\left(q^{4}+q^{3}+q^{2}+q+1\right)$, and $k$ divides $2 q\left(q^{2}+q+1\right)$. The fact that $k^{2}>2 v$ forces $k=2 q\left(q^{2}+q+1\right)$.

The condition $k(k-1)=2(v-1)$ implies

$$
4 q^{2}\left(q^{2}+q+1\right)^{2}-2 q\left(q^{2}+q+1\right)=2\left(q^{6}+q^{5}+2 q^{4}+2 q^{3}+2 q^{2}+q\right),
$$

so

$$
\left(q^{2}+q+1\right)\left(2 q\left(q^{2}+q+1\right)-1\right)=\left(q^{5}+q^{4}+2 q^{3}+2 q^{2}+2 q+1\right) .
$$

If we expand we get the following equality:

$$
q^{5}+3 q^{4}+4 q^{3}+q^{2}-q-2=0,
$$

which is a contradiction. Therefore $n \geq 7$. Here

$$
v=\left(q^{n-1}+q^{n-2}+\cdots+q+1\right)\left(q^{n-3}+q^{n-5}+\cdots+q^{2}+1\right),
$$

and $k$ divides $2 d c$, where $d=q\left(q^{n-3}+q^{n-4}+\cdots+q+1\right)$ and $c=\left(q+1, \frac{n-3}{2}\right)$. Say $k=\frac{2 d c}{e}$, then $v<k^{2}$ forces $e \leq 2 q$. We have the following equality:

$$
\frac{v-1}{d}=q^{n-2}+q^{n-4}+\cdots+q^{3}+q+1,
$$

and also, since $k(k-1)=2(v-1)$, we have

$$
k=\frac{2(v-1)}{k}+1=\frac{2 e(v-1)}{2 d c}=\frac{e q^{n-2}+e q^{n-4}+\cdots+e q^{3}+e q+e+c}{c} .
$$


Now, $(k c, d)$ divides $d$, and also

$\left(k c, q\left(e q^{n-3}+e q^{n-5}+\cdots+e q^{2}+e\right)\right)$

$=\left(e q^{n-2}+e q^{n-4}+\cdots+e q+e+c, q\left(e q^{n-3}+e q^{n-5}+\cdots+e q^{2}+e\right)\right)$

$=\left(e q^{n-2}+\cdots+e q+e+c, e+c\right)$, and

$\left(k c, \frac{e d}{q}\right)$

$=\left(e q^{n-2}+\cdots+e q+e+c, e q^{n-3}+e q^{n-4}+\cdots+e q+e\right)$

$=\left(e q^{n-2}+\cdots+e q+e+c,(2 e+c) q+e+c\right)$.

Therefore $k$ divides $c(e+c)((2 e+c) q+e+c)$, and since $e \leq 2 q$ and $c=$ $\left(q+1, \frac{n-3}{2}\right)$, the only possibilities for $n$ and $q$ are $n=7$ and $q \leq 3$, or $n=9$ and $q=2$. However in none of these possibilities is $8 v-7$ a square, again contradicting Lemma 2.

$\left.\mathcal{C}_{1}^{\prime}\right)$ Here $G$ contains a graph automorphism and $G_{x}$ stabilises a pair $\{U, W\}$ of subspaces of dimension $i$ and $n-i$, with $i<\frac{n}{2}$. Write $G^{0}$ for $G \cap P \Gamma L_{n}(q)$ of index 2 in $G$.

First assume $U \subset W$. By Lemma 10, there is a subdegree which is a power of $p$. On the other hand, if $p$ is odd then the highest power of $p$ dividing $v-1$ is $q$, it is $2 q$ if $q>2$ is even, and is at most $2^{n-1}$ if $q=2$. Hence $k^{2}<v$, which is a contradiction.

Now suppose $V=U \oplus W$. Here $p$ divides $v$, so $(k, p) \leq 2$. First assume $i=1$. If $x=\left\{\left\langle v_{1}\right\rangle,\left\langle v_{2} \ldots v_{n}\right\rangle\right\}$, then consider $y=\left\{\left\langle v_{1}, \ldots, v_{n-1}\right\rangle,\left\langle v_{n}\right\rangle\right\}$, so $\left[G_{x}: G_{x y}\right]=$ $\frac{q^{n-2}\left(q^{n-1}-1\right)}{q-1}$ and $k$ divides $\frac{2\left(q^{n-1}-1\right)}{q-1}$. However $v=\frac{q^{n-1}\left(q^{n}-1\right)}{q-1}>q^{2(n-1)}$, which implies $k^{2}<v$, a contradiction.

Now assume $i>1$. Consider $x=\left\{\left\langle v_{1}, \ldots, v_{i}\right\rangle,\left\langle v_{i+1}, \ldots, v_{n}\right\rangle\right\}$ and $y=\left\{\left\langle v_{1}, \ldots\right.\right.$, $\left.\left.v_{i-1}, v_{i}+v_{n}\right\rangle,\left\langle v_{i+1}, \ldots, v_{n}\right\rangle\right\}$. Then $\left[G_{x}^{0}: G_{x y}^{0}\right]_{p^{\prime}}$ divides $2\left(q^{i}-1\right)\left(q^{n-i}-1\right)$, which implies $k<2 q^{n}$, but $v>q^{2 i(n-i)}$, so again $k^{2}<v$, a contradiction.

$\mathcal{C}_{2}$ ) Here $G_{x}$ preserves a partition $V=V_{1} \oplus \cdots \oplus V_{a}$, with each $V_{i}$ of the same dimension, say, $b$, and $n=a b$.

First consider the case $b=1$ and $n=a$, and let $x=\left\{\left\langle v_{1}\right\rangle, \ldots,\left\langle v_{n}\right\rangle\right\}$ and $y=$ $\left\{\left\langle v_{1}+v_{2}\right\rangle,\left\langle v_{2}\right\rangle, \ldots,\left\langle v_{n}\right\rangle\right\}$. Since $n>2$, we see $k$ divides $4 n(n-1)(q-1)=$ $2\left[G_{x}: G_{x y}\right]$. Now $v>\frac{q^{n(n-1)}}{n !}$ and $k^{2}>v$, so $n=3$ and $q \leq 4$, that is $v=$ $\frac{q^{3}\left(q^{3}-1\right)(q+1)}{(3, q-1) 6 !}$. As $k \mid 2(v-1)$, only for $q=2$ can $k>2$, so consider $q=2$. Then $k \mid 6$ and $v=28$, but there is no such value of $k$ satisfying $k(k-1)=2(v-1)$.

Now let $b>1$, and consider $x=\left\{\left\langle v_{1}, \ldots, v_{b}\right\rangle,\left\langle v_{b+1}, \ldots, v_{2 b}\right\rangle, \ldots\right\}$ and $y=$ $\left\{\left\langle v_{1}, \ldots, v_{b-1}, v_{b+1}\right\rangle,\left\langle v_{b}, v_{b+2}, \ldots, v_{2 b}\right\rangle, \ldots,\left\langle v_{n-b+1}, \ldots, v_{n}\right\rangle\right\}$. Then $k$ divides $\frac{2 a(a-1)\left(q^{b}-1\right)^{2}}{q-1}$, so $v>\frac{q^{n(n-b)}}{a !}$, forcing $n=4, q \geq 5$, and $a=2=b$. In none of these cases can we obtain $k>2$.

$\mathcal{C}_{3}$ ) In this case $G_{x}$ is an extension field subgroup. Since $2\left|G_{x}\right|\left|G_{x}\right|_{p^{\prime}}^{2}>|G|$ by Corollary 8, either:

(1) $n=3$ and $X \cap G_{x}={ }^{\wedge}\left(q^{2}+q+1\right) \cdot 3<P S L_{3}(q)=X$, or

(2) $n$ is even and $G_{X}=N_{G}\left({ }^{\wedge} P S L_{\frac{n}{2}}\left(q^{2}\right)\right)$.

First consider case (1). Here $v=\frac{q^{3}\left(q^{2}-1\right)(q-1)}{3}$, so $k$ divides $6\left(q^{2}+q+1\right)\left(\log _{p} q\right)$, and $k^{2}>v$ implies $q=3,4,5,8,9,11,13$, or 16 . In none of these cases is $8 v-7$ a square. 
Now consider case (2) and write $n=2 m$. As $p$ divides $v$, we have $(k, p) \leq 2$. First suppose $n \geq 8$, and let $W$ be a 2-subspace of $V$ considered as a vector space over the field of $q^{2}$ elements, so that $W$ is a 4-subspace over a field of $q$ elements. If we consider the stabiliser of $W$ in $G_{x}$ and in $G$ then in $G_{W} \backslash G_{x W}$ there is an element $g$ such that $G_{x} \cap G_{x}^{g}$ contains the pointwise stabiliser of $W$ in $G_{x}$ as a subgroup. Therefore $k$ divides $2\left(q^{n}-1\right)\left(q^{n-2}-1\right)$, contrary to $2 v<k^{2}$, which is a contradiction.

Now let $n=6$. Then since $(k, p) \leq 2$, Lemma 9 implies $k$ is divisible by the index of a parabolic subgroup of $G_{x}$, so it is divisible by the primitive prime divisor $q_{3}$ of $q^{3}-1$, but this divides the index of $G_{x}$ in $G$, which is $v$, a contradiction.

Hence $n=4$. Then $v=\frac{q^{4}\left(q^{3}-1\right)(q-1)}{2}$, and so $k$ is odd and prime to $q-1$. The fact that $(v-1, q+1)=1$ implies $k$ is also prime to $q+1$, and hence $k \mid\left(q^{2}+1\right) \log _{p} q$, contrary to $k^{2}>2 v$, another contradiction.

$\mathcal{C}_{4}$ ) Here $G_{x}$ stabilises a tensor product of spaces of different dimensions, and $n \geq 6$. In all these cases $v>k^{2}$.

$\mathcal{C}_{5}$ ) In this case $G_{x}$ is the stabiliser in $G$ of a subfield space. So $G_{x}=$ $N_{G}\left(P S L_{n}\left(q_{0}\right)\right)$, with $q=q_{0}^{m}$ and $m$ prime.

If $m>2$ then $2\left|G_{x}\right|\left|G_{x}\right|_{p^{\prime}}^{2}>|G|$ forces $n=2$, a contradiction.

Hence $m=2$. If $n=3$ then $v=\frac{\left(q_{0}^{3}+1\right)\left(q_{0}^{2}+1\right) q_{0}^{3}}{\left(q_{0}+1,3\right)}$.

Since $p$ divides $v$, we have $(k, p) \stackrel{2}{\leq}$, so Lemma 9 implies $G_{x B}$ (where $B$ is a block incident with $x$ ) is contained in a parabolic subgroup of $G_{x}$. Therefore $q_{0}^{2}+$ $q_{0}+1$ divides $k$, and $\left(v-1, q_{0}^{2}+q_{0}+1\right)$ divides $2 q_{0}+\left(q_{0}+1,3\right)$, forcing $q_{0}=2$ and $v=120$, but then $8 v-7$ is not a square.

If $n=4$, then by Lemma 9 we see $q_{0}^{2}+1$ divides $k$, but $q_{0}^{2}+1$ also divides $v$, which is a contradiction.

Hence $n \geq 5$. Considering the stabilisers of a 2-dimensional subspace of $V$, we see $k$ divides $2\left(q_{0}^{n}-1\right)\left(q_{0}^{n-1}-1\right)$, but then $k^{2}<v$, which is also a contradiction.

$\mathcal{C}_{6}$ ) Here $G_{x}$ is an extraspecial normaliser. Since $2\left|G_{x}\right|\left|G_{x}\right|_{p^{\prime}}^{2}>|G|$, we have $n \leq$ 4. Now, $n>2$ implies that $G_{x} \cap X$ is either $2^{4} A_{6}$ or $3^{2} Q_{8}$, with $X$ either $P S L_{4}(5)$ or $P S L_{3}(7)$ respectively. Since $k$ divides $2\left(v-1,\left|G_{x}\right|\right)$, we check that $k \leq 6$, contrary to $k^{2}>2 v$.

If $n=2$ then $G_{x} \cap X=A_{4} . a<L_{2}(p)=X$, with $a=2$ precisely when $p \equiv \pm 1$ $(\bmod 8)$, and $a=1$ otherwise, (and there are $a$ conjugacy classes in $X)$. From $|G|<$ $\left|G_{x}\right|^{3}$ we obtain $p \leq 13$. If $p=7$ then the action is 2 -transitive. The remaining cases are ruled out by the fact that $k$ divides $2\left(v-1,\left|G_{x}\right|\right)$, and $k(k-1)=2(v-1)$.

$\mathcal{C}_{7}$ ) Here $G_{x}$ stabilises the tensor product of $a$ spaces of the same dimension, say $b$, and $n=b^{a}$. Since $\left|G_{x}\right|^{3}>|G|$, we have $n=4$ and $G_{x} \cap X=\left(P S L_{2}(q) \times\right.$ $\left.P S L_{2}(q)\right) 2^{d}<X=P S L_{4}(q)$, with $d=(2, q-1)$. Then $v=\frac{q^{4}\left(q^{2}+1\right)\left(q^{3}-1\right)}{x}>\frac{q^{9}}{x}$, with $x=2$ unless $q \equiv 1(\bmod 4)$, in which case $x=4$. Hence $4 \nmid k$, and so $k$ divides $2\left(q^{2}-1\right) \log _{p} q$, and if $q$ is odd then $k$ divides $\frac{\left(q^{2}-1\right) \log _{p} q}{32}$.

If $q$ is odd, then $k^{2}<\frac{q^{9}}{32}<\frac{q^{9}}{x}=v$, a contradiction. Hence $q$ is even, and so

$$
k=\frac{2\left(q^{2}-1\right)^{2} \log _{p} q}{r},
$$


and since $k^{2}>2 v$ we have $r^{2}<\frac{4(q+1)^{4} \log _{p} q}{q^{5}}$, therefore $q \leq 32$.

However, the five cases are dismissed by the fact that $k$ divides $2(v-1)$.

$\mathcal{C}_{8}$ ) Now consider $G_{x}$ to be a classical group.

(1) First assume $G_{x}$ is a symplectic group, so $n$ is even. By Lemma $6 k$ is divisible by a parabolic index in $G_{x}$. If $n=4$ then $v=\frac{q^{2}\left(q^{3}-1\right)}{(2, q-1)}$, and $\frac{q^{4}-1}{q-1}$ divides $k$, however $\left(v-1, q^{2}+1\right)$ divides 2 , which is a contradiction.

If $n=6$ then $v=\frac{q^{6}\left(q^{5}-1\right)\left(q^{3}-1\right)}{(3, q-1)}$ and $q^{3}+1$ divides $k$, but $q^{3}+1$ divides $2(v-1)$ only if $q=2$, so $k=9$, too small.

Now suppose $n \geq 8$. If we consider the stabilisers of a 4-dimensional subspace of $G_{x}$ and $G$, we see that $k$ divides twice the odd part of $\left(q^{n}-1\right)\left(q^{n-2}-1\right)$. Also, $(k, q-1) \leq 2$, so $k$ divides $2 \frac{\left(q^{n}-1\right)\left(q^{n-2}-1\right)}{(q-1)^{2}}$, and therefore $k \leq 8 q^{2 n-4}$. The inequality $k^{2}>2 v$ forces $n=8$. In this case $v=\frac{q^{12}\left(q^{7}-1\right)\left(q^{5}-1\right)\left(q^{3}-1\right)}{(q-1,4)}$ which implies $q \leq 3$, and in neither of these two cases is $8 v-7$ a square.

(2) Now let $G_{x}$ be orthogonal. Then $q$ is odd, since that is the case with odd dimension, and with even dimension it is a consequence of the maximality of $G_{x}$ in $G$. The case in which $n=4$ and $G_{x}$ is of type $O_{4}^{+}$will be investigated later, in all other cases Lemma 6 implies that $k$ is divisible by a parabolic index in $G_{x}$ and is therefore even, but it is not divisible by 4 since $v$ is also even and $(k, v) \leq 2$. This and the fact that $q$ does not divide $k$ implies $k<v$, a contradiction.

(3) Finally let $G_{x}$ be a unitary group over the field of $q_{0}$ elements, where $q=q_{0}^{2}$. If $n \geq 4$ then considering the stabilisers of a nonsingular 2-subspace of $V$ in $G$ and $G_{x}$, we see $k$ divides $2\left(q_{0}^{n}-(-1)^{n}\right)\left(q_{0}^{n-1}-(-1)^{n-1}\right)$. The inequality $k^{2}>2 v$ forces $n=4$, and in this case $v=\frac{q_{0}^{6}\left(q_{0}^{4}+1\right)\left(q_{0}^{3}+1\right)\left(q_{0}^{2}+1\right)}{\left(q_{0}-1,4\right)}$. Since $k$ divides $2\left(q_{0}^{4}-1\right)\left(q_{0}^{3}+1\right)$ and $\left(k,\left(q_{0}^{2}+1\right)\left(q_{0}-1\right) \leq 2\right.$, we see $k$ divides $2\left(q_{0}^{3}+1\right)\left(q_{0}+1\right)$, so $k^{2} \leq 2 v$, a contradiction. Therefore $n=3$, and by Lemma $6 q_{0}^{2}-q_{0}+1$ divides $k$, and $k$ divides $2(v-1)$ with $v=\frac{q_{0}^{3}\left(q_{0}^{3}-1\right)\left(q_{0}^{2}+1\right)}{x}$ with $x$ either 1 or 3 . This implies $q_{0}=2$, but then $v=280$, and $8 v-7$ is not a square.

$\mathcal{S}$ ) We finally consider the case where $G_{x}$ is an almost simple group, (modulo the scalars), not contained in the Aschbacher subgroups of $G$. From [18, Theorem 4.2] we have the possibilities $\left|G_{x}\right|<q^{2 n+4}, G_{x}^{\prime}=A_{n-1}$ or $A_{n-2}$, or $G_{x} \cap X$ and $X$ are as in [18, Table 4].

Also, $|G|<\left|G_{x}\right|^{3}$ by Corollary 3 and $|G| \leq q^{n^{2}-n-1}$, so $n \leq 7$, and by the bound $2\left|G_{x}\right|\left|G_{x}\right|_{p^{\prime}}^{2}>|G|$ we need only to consider the following possibilities [15, Chapter 5]:

$n=2$, and $G_{X} \cap X=A_{5}$, with $q=11,19,29,31,41,59,61$, or 121.

$n=3$, and $G_{x} \cap X=A_{6}<P S L_{3}(4)=X$.

$n=4$, and $G_{X} \cap X=U_{4}(2)<P S L_{4}(7)=X$.

In the first case, with $A_{5}<L_{2}(11)$ the action is 2-transitive. In the remaining cases, the fact that $k$ divides $2\left|G_{x}\right|$ and $2(v-1)$ forces $k^{2}<v$, which is a contradiction.

This completes the proof of Lemma 12 . 


\section{$4 X$ is a symplectic group}

Here the socle of $G$ is $X=P \operatorname{Sp}_{2 m}(q)$, with $m \geq 2$ and $(m, q) \neq(2,2)$. As a standard symplectic basis for $V$, we have $\beta=\left\{e_{1}, f_{1}, \ldots, e_{m}, f_{m}\right\}$.

Lemma 13 The group $X$ is not $P \operatorname{Sp}_{2 m}(q)$ with $m \geq 2$, and $(m, q) \neq(2,2)$.

Proof We will consider $G_{x}$ to be in each of the Aschbacher families of subgroups, and finally, an almost simple group not contained in any of the Aschbacher families of $G$. In each case we will arrive at a contradiction.

When $(p, 2 m)=(2,4)$ the group $S p_{4}\left(2^{f}\right)$ admits a graph automorphism, this case will be treated separately after the eight Aschbacher families of subgroups.

$\mathcal{C}_{1}$ ) If $G_{x} \in \mathcal{C}_{1}$, then $G_{x}$ is reducible, so either it is parabolic or it stabilises a nonsingular subspace of $V$.

First assume that $G_{x}=P_{i}$, the stabiliser of a totally singular $i$-subspace of $V$, with $i \leq m$. Then

$$
v=\frac{\left(q^{2 m}-1\right)\left(q^{2 m-2}-1\right) \ldots\left(q^{2 m-2 i+2}-1\right)}{\left(q^{i}-1\right)\left(q^{i-1}-1\right) \ldots(q-1)} .
$$

From this we see $v \equiv q+1(\bmod p q)$, so $q$ is the highest power of $p$ dividing $v-1$. By Lemma 10 there is a subdegree which is a power of $p$, and since $k$ divides twice every subdegree, $k$ divides $2 q$, contrary to $v<k^{2}$.

Now suppose that $G_{x}=N_{2 i}$, the stabiliser of a nonsingular $2 i$-subspace $U$ of $V$, with $m>2 i$. Then $p$ divides $v$, so $(k, p) \leq 2$.

Take $U=\left\langle e_{1}, f_{1}, \ldots e_{i}, f_{i}\right\rangle$, and $W=\left\langle e_{1}, f_{1}, \ldots e_{i-1}, f_{i-1}, e_{i+1}, f_{i+1}\right\rangle$. The $p^{\prime}-$ part of the size of the $G_{x}$-orbit containing $W$ is

$$
\frac{\left(q^{2 i}-1\right)\left(q^{2 m-2 i}-1\right)}{\left(q^{2}-1\right)^{2}} .
$$

Since $v<q^{4 i(m-i)}$, we can only have $v<k^{2}$ if $q=2$ and $m=i+1$, which is a contradiction.

$\mathcal{C}_{2}$ ) If $G_{x} \in \mathcal{C}_{2}$ then it preserves a partition $V=V_{1} \oplus \cdots \oplus V_{a}$ of isomorphic subspaces of $V$.

First assume all the $V_{j}$ 's to be totally singular subspaces of $V$ of maximal dimension $m$. Then $G_{x} \cap X=\hat{\wedge} G L_{m}(q) .2$, and $G_{x}$ maximal implies $q$ is odd [17]. Then

$$
v=\frac{q^{\frac{m(m+1)}{2}}\left(q^{m}+1\right)\left(q^{m-1}+1\right) \ldots(q+1)}{2}>\frac{q^{m(m+1)}}{2},
$$

and $(k, p)=1$.

Let

$$
x=\left\{\left\langle e_{1}, \ldots, e_{m}\right\rangle,\left\langle f_{1}, \ldots, f_{m}\right\rangle\right\},
$$

and

$$
y=\left\{\left\langle e_{1}, \ldots, e_{m-1}, f_{m}\right\rangle,\left\langle f_{1}, \ldots, f_{m-1}, e_{m}\right\rangle\right\} .
$$


Then the $p^{\prime}$-part of the $G_{x}$-orbit of $y$ divides $2\left(q^{m}-1\right)$, and so $k$ divides $4\left(q^{m}-1\right)$, contrary to $v<k^{2}$.

Now assume that each of the $V_{j}$ 's is nonsingular of dimension $2 i$, so $G_{x} \cap X=$ $\hat{S} p_{2 i}(q)$ wr $S_{t}$, with $i t=m$. Let

$$
x=\left\{\left\langle e_{1}, f_{1}, \ldots, e_{i}, f_{i}\right\rangle,\left\langle e_{i+1}, f_{i+1}, \ldots, e_{2 i}, f_{2 i}\right\rangle, \ldots\right\},
$$

and take

$$
y=\left\{\left\langle e_{1}, f_{1}, \ldots, e_{i}, f_{i}+e_{i+1}\right\rangle,\left\langle e_{i+1}, f_{i+1}-e_{i}, e_{i+2}, \ldots, e_{2 i}, f_{2 i}\right\rangle, \ldots\right\} .
$$

Considering the size of the $G_{x}$-orbit containing $y$, we see $k$ divides

$$
\frac{t(t-1)\left(q^{2 i}-1\right)^{2}}{q-1} .
$$

Now,

$$
\frac{q^{2 i^{2} t(t-1)}}{t !}<v,
$$

so $v<k^{2}$ implies $t ! t^{4}>q^{2 i^{2} t(t-1)+2-8 i}$, hence $q^{2 t(t-1)-6}<t^{t+4}$ and therefore $t<4$.

First assume $t=3$. Then by the above inequalities $i=1$ and $q=2$, but then $G_{x}$ is not maximal [8, p. 46], a contradiction.

Now let $t=2$. Then $k<2 q^{4 i-1}$, so $q^{4 i^{2}-8 i+2}<8$ and therefore $i \leq 2$.

If $i=2$ then $q=2$ and $v=45696=2^{7} \cdot 3 \cdot 7 \cdot 17$, but then $8 v-7$ is not a square, which is a contradiction.

If $i=1$ then $X=P S p_{4}(q)$,

$$
v=\frac{q^{2}\left(q^{2}+1\right)}{2}
$$

and $k$ divides $2(q+1)^{2}(q-1)$. Since $k$ divides $2(v-1)$, we have $k$ divides $\left(q^{2}\left(q^{2}+\right.\right.$ 1) $\left.-2,2(q+1)^{2}(q-1)\right)$, that is, $k$ divides

$$
\left(\left(q^{2}+2\right)\left(q^{2}-1\right), 2(q+1)^{2}(q-1)\right)=\left(q^{2}-1\right)\left(q^{2}+2,2(q+1)\right) \leq 6\left(q^{2}-1\right) .
$$

Therefore

$$
k=\frac{6\left(q^{2}-1\right)}{r},
$$

with $1 \leq r \leq 6$. Now $2(v-1)=\left(q^{2}+2\right)\left(q^{2}-1\right)$, and also $2(v-1)=k(k-1)$, but we check that for all possible values of $r$ this equality is not satisfied.

$\mathcal{C}_{3}$ ) If $G_{x} \in \mathcal{C}_{3}$, then it is an extension field subgroup, and there are two possibilities.

Assume first that $G_{x} \cap X=P S p_{2 i}\left(q^{t}\right) . t$, with $m=i t$ and $t$ a prime number. From $|G|<\left|G_{x}\right|^{3}$, we obtain $t=2$ or 3 .

If $t=3$, then $v<k^{2}$ implies $i=1$, and so

$$
G_{x} \cap X=P \operatorname{Sp}_{2}\left(q^{3}\right)<P \operatorname{Sp}_{6}(q)=X,
$$


and

$$
v=\frac{q^{6}\left(q^{4}-1\right)\left(q^{2}-1\right)}{3} .
$$

This implies that $k$ is coprime to $q+1$, but applying Lemma 9 to $P \operatorname{Sp}_{2}\left(q^{3}\right)$ yields $q^{3}+1$ divides $k$, which is a contradiction.

If $t=2$, then

$$
v=\frac{q^{2 i^{2}}\left(q^{4 i-2}-1\right)\left(q^{4 i-6}-1\right) \ldots\left(q^{6}-1\right)\left(q^{2}-1\right)}{2} .
$$

Consider the subgroup $S p_{2}\left(q^{2}\right) \circ S p_{2 i-2}\left(q^{2}\right)$ of $G_{x} \cap X$. This is contained in $S p_{4}(q) \circ$ $S p_{4 i-4}(q)$ in $X$. Taking $g \in S p_{4}(q) \backslash S p_{2}\left(q^{2}\right)$, we see $S p_{2 i-2}\left(q^{2}\right)$ is contained in $G_{x} \cap G_{x}^{g}$, so $k$ divides $2\left(q^{4 i}-1\right) \log _{p} q$. The inequality $v<k^{2}$ forces $i \leq 2$.

First assume $i=2$. Then

$$
v=\frac{q^{8}\left(q^{6}-1\right)\left(q^{2}-1\right)}{2}
$$

and $k$ divides $2\left(q^{8}-1\right) \log _{p} q$, but since $(k, v) \leq 2$ and $q^{2}-1$ divides $v$, we see $k$ divides $2\left(q^{4}+1\right)\left(q^{2}+1\right) \log _{p} q$, forcing $q=2$. In this case $v=2^{7} \cdot 3^{3} \cdot 7=24192$, and $k=2 \cdot 5 \cdot 17=170$ (otherwise $k^{2}<v$ ), but then $k$ does not divide $2(v-1$ ), which is a contradiction.

Hence $i=1$, so

$$
v=\frac{q^{2}\left(q^{2}-1\right)}{2}
$$

and $G_{x} \cap X=P \operatorname{Sp}_{2}\left(q^{2}\right) .2<P \operatorname{Sp}_{4}(q)=X$, Therefore $k$ divides $4 q^{2}\left(q^{4}-1\right)$, but since $(k, v) \leq 2$, then $k$ divides $4\left(q^{2}+1\right)$, so $k=\frac{4\left(q^{2}+1\right)}{r}$ for some $r \leq 8$ (since $\left.v<k^{2}\right)$. Now $2(v-1)=k(k-1)$, and also $2(v-1)=\left(q^{2}-2\right)\left(q^{2}+1\right)$, so we have

$$
r^{2}\left(q^{2}-2\right)=16\left(q^{2}+1\right)-4 r
$$

that is,

$$
(r+4)(r-4) q^{2}=2(8+r(r-2)) .
$$

This implies $4<r \leq 8$, but solving the above equation for each of these possible values of $r$ gives non-integer values of $q$, a contradiction.

Now assume $G_{X} \cap X=\hat{}{ }^{\wedge} U_{m}(q) .2$, with $q$ odd. Since $v$ is even, 4 does not divide $k$. Also, $k$ is prime to $p$, so by the Lemma 9 , the stabiliser in $G_{x} \cap X$ of a block is contained in a parabolic subgroup. But then $q+1$ divides the indices of the parabolic subgroups in the unitary group, so $q+1$ divides $k$, but $q+1$ also divides $v$, which is a contradiction.

$\mathcal{C}_{4}$ ) If $G_{x} \in \mathcal{C}_{4}$, then $G_{x}$ stabilises a decomposition of $V$ as a tensor product of two spaces of different dimensions, and $G_{x}$ is too small to satisfy

$$
|G|<2\left|G_{X}\right|\left|G_{X}\right|_{p^{\prime}}^{2} .
$$


$\mathcal{C}_{5}$ ) If $G_{x} \in \mathcal{C}_{5}$, then $G_{x} \cap X=P \operatorname{Sp}_{2 m}\left(q_{0}\right) . a$, with $q=q_{0}^{b}$ for some prime $b$ and $a \leq 2$, (with $a=2$ if and only if $b=2$ and $q$ is odd). The inequality $|G|<$ $2\left|G_{x}\right|\left|G_{x}\right|_{p^{\prime}}^{2}$ forces $b=2$. Then

$$
v=\frac{q^{\frac{m^{2}}{2}}\left(q^{m}+1\right) \ldots(q+1)}{(2, q-1)}>\frac{q^{\frac{m(2 m+1)}{2}}}{2} .
$$

Now $G_{x}$ stabilises a $G F\left(q_{0}\right)$-subspace $W$ of $V$. Considering a nonsingular 2-dimensional subspace of $W$ we see

$$
S p_{2}\left(q_{0}\right) \circ S p_{2 m-2}\left(q_{0}\right)<S p_{2}(q) \circ S p_{2 m-2}(q)<X .
$$

If we take $g \in S p_{2}(q) \backslash S p_{2}\left(q_{0}\right)$ then $S p_{2 m-2}\left(q_{0}\right)<G_{x} \cap G_{x}^{g}$. This implies that there is a subdegree of $X$ with the $p^{\prime}$-part dividing $q_{0}^{2 m}-1$, so $k$ divides $2\left(q^{m}-\right.$ 1) $\log _{p} q$, contrary to $v<k^{2}$.

$\mathcal{C}_{6}$ ) If $G_{x} \in \mathcal{C}_{6}$ then $G_{x} \cap X=2^{2^{s}} \Omega_{2^{s}}^{-}(2)$. $a, q$ is an odd prime, $2 m=2^{s}$, and $a \leq 2$. The inequality $|G|<\left|G_{x}\right|^{3}$ implies $s \leq 3$, and if $s=3$ then $q=3$, but then $k$ is too small. If $s=2$ then $q \leq 11$, but again $k$ is too small in each of these cases.

$\left.\mathcal{C}_{7}\right)$ If $G_{x} \in \mathcal{C}_{7}$ then $G_{x}=N_{G}\left(P S p_{2 a}(q)^{2 r} 2^{r-1} A_{r}\right)$ and $2 m=(2 a)^{r} \geq 8$, but this is a contradiction since $|G|<\left|G_{x}\right|^{3}$.

$\left.\mathcal{C}_{8}\right)$ If $G_{x} \in \mathcal{C}_{8}$ then $G_{x} \cap X=O_{2 m}^{\epsilon}(q)$, with $q$ even and $2 m \geq 4$. We can assume $q>2$ as when $q=2$ the action is 2-transitive and that has been done in [14]. Here

$$
v=\frac{q^{m}\left(q^{m}+\epsilon\right)}{2}
$$

and from the proof of [23, Prop. 1] the subdegrees of $X$ are $\left(q^{m}-\epsilon\right)\left(q^{m+1}+\epsilon\right)$ and $\frac{(q-2)}{2} q^{m-1}\left(q^{m}-\epsilon\right)$. This implies by Lemma 4 that $k$ divides $2\left(q^{m}-\epsilon\right)(q-$ $\left.2, q^{m-1}+\epsilon\right)$. However, Lemma 9 implies $k$ is divisible by the index of a parabolic subgroup in $O_{2 m}^{\epsilon}(q)$, which is not the case.

$p=m=2$. Here $2 m=4$ and $q$ is even, we have the following possibilities:

$G_{x}$ normalises a Borel subgroup of $X$ in $G$. Then $v=(q+1)\left(q^{3}+q^{2}+q+1\right)$ so $2 q$ is the highest power of 2 dividing $v-1$. But $k$ is also a power of 2 , contrary to $v<k^{2}$.

$G_{X} \cap X=D_{2(q \pm 1)}$ wr $S_{2}$. So $k$ divides $2(q \pm 1)^{2} \log _{2} q$, too small to satisfy $v<k^{2}$. $G_{x} \cap X=\left(q^{2}+1\right) .4$, which is too small.

$\mathcal{S})$ Finally consider the case in which $G_{x} \in \mathcal{S}$ is an almost simple group (modulo scalars) not contained in any of the Aschbacher subgroups of $G$. These subgroups are listed in [15] for $2 m \leq 10$.

First assume $2 m=4$, so we have one of the following possibilities:

(1) $G_{X} \cap X=S z(q)$ with $q$ even,

(2) $G_{X} \cap X=P S L_{2}(q)$ with $q \geq 5$, or

(3) $G_{x} \cap X=A_{6} . a$ with $a \leq 2$ and $q=p \geq 5$.

In case (1) $v=q^{2}\left(q^{2}-1\right)(q+1)$. Applying Lemma 9 to $S z(q)$, we see $q^{2}+1$ divides $k$. Now $\left(v-1, q^{2}+1\right)=(q-2,5)$, so $q=2$, contrary to our initial assumptions. 
In case (2), since $(k, v) \leq 2$, we have $k \leq 2 \log _{p} q$, contrary to $v<k^{2}$.

In case (3), 4 does not divide $k$, so $k$ must divide 90, contrary to $v<k^{2}$.

Now let $2 m=6$. As $|G|<2\left|G_{x}\right|\left|G_{X}\right|_{p^{\prime}}^{2}$, from [15] either $G_{x} \cap X=J_{2}<$ $P \operatorname{Sp}_{6}(5)=X$, or $G_{X} \cap X=G_{2}(q)$ with $q$ even. In the first case $k$ divides $2 \cdot 3^{3} \cdot 7$, which is too small. In the second case $v=q^{3}\left(q^{4}-1\right) 4$, so $(k, q+1)=1$. Applying Lemma 9 to $G_{2}(q)$ we see that $\frac{q^{6}-1}{q-1}$ divides $k$, a contradiction.

If $2 m=8$ or 10 , then by [15] either $G_{x}=S_{10}<S p_{8}(2)=G$ or $G_{x}=S_{14}<$ $S p_{12}(2)=G$. In the first case $k$ divides $2\left(v-1,\left|G_{x}\right|\right)=70$, which is too small. In the second case $(k, v) \leq 2$ implies that $k$ divides $2 \cdot 7^{2} \cdot 11 \cdot 13$, also too small.

If $2 m \geq 12$, then by [18] we have $\left|G_{x}\right| \leq q^{4(m+1)}, G_{x}^{\prime}=A_{n+1}$ or $A_{n+2}$, or $X$ or $G_{x} \cap X$ are $E_{7}(q) \leq P \operatorname{Sp}_{56}(q)$. The latter is not possible as here $k^{2}<v$, and the bound $\left|G_{x}\right|<q^{4(m+1)}$ forces $m<6$.

The only possibilities for the alternating groups are $q=2$, and $m=7,8$, or 9 , however in all these cases $k$ is too small.

This completes the proof of Lemma 13.

\section{$5 X$ is an orthogonal group of odd dimension}

Here we consider $X=P \Omega_{2 m+1}(q)$, with $q$ odd and $n=2 m+1 \geq 7$, (since $\Omega_{3}(q) \cong$ $L_{2}(q)$, and $\left.\Omega_{5}(q) \cong P S p_{4}(q)\right)$.

Lemma 14 The group $X$ is not $P \Omega_{2 m+1}(q)$, with $n \geq 7$.

Proof Here, as in the symplectic case, we will consider $G_{x}$ to be in each of the Aschbacher families of subgroups, and then to be a subgroup of $G$ not contained in any of these families, and arrive at a contradiction in each case.

$\mathcal{C}_{1}$ ) If $G_{x} \in \mathcal{C}_{1}$, then $G_{x}$ is either parabolic or it stabilises a nonsingular subspace of $V$.

First assume $G_{x}=P_{i}$, the stabiliser of a totally singular $i$-subspace of $V$. Then, as in the symplectic case, $v \equiv q+1(\bmod p q)$, so $q$ is the highest power of $p$ dividing $v-1$. By Lemma 10 there is a subdegree which is a power of $p$, therefore $k$ divides $2 q$, contradicting $v<k^{2}$.

Now assume that $G_{x}=N_{i}^{\epsilon}$, the stabiliser of a nonsingular $i$-dimensional subspace $W$ of $V$ of sign $\epsilon$ (if $i$ is odd $\epsilon$ is the sign of $W^{\perp}$ ).

First let $i=1$. Then

$$
v=\frac{q^{m}\left(q^{m}+\epsilon\right)}{2}
$$

and the $X$-subdegrees are $\left(q^{m}-\epsilon\right)\left(q^{m}+\epsilon\right), \frac{q^{m-1}\left(q^{m}-\epsilon\right)}{2}$, and $\frac{q^{m-1}\left(q^{m}-\epsilon\right)(q-3)}{2}$. This implies that $k$ divides $q^{m}-\epsilon$, contrary to $v<k^{2}$.

Hence $i \geq 2$. Let $W$ be the $i$-space stabilised by $G_{x}$ and choose $w \in W$ with $\mathcal{Q}(w)=1$, and $u \in W^{\perp}$ with $\mathcal{Q}(u)=-c$ for some non-square $c \in G F(q)$. Then $\langle v, w\rangle$ is of type $N_{2}^{-}$, and if $g \in G$ stabilises $W^{\perp}$ pointwise but does not fix neither $u$ 
nor $w$, then $G_{x} \cap G_{x}^{g}$ contains $S O_{i-1}(q) \times S O_{n-i-1}(q)$. This implies $k \leq 4 q^{m} \log _{p} q$, but $v>q^{\frac{i(n-i)}{4}}$ implies $q$ is odd and $m \geq 3$, this is contrary to $v<k^{2}$.

$\mathcal{C}_{2}$ ) If $G_{x} \in \mathcal{C}_{2}$ then $G_{x}$ is the stabiliser of a subspace decomposition into isometric nonsingular spaces. From the inequality $|G|<2\left|G_{x}\right|\left|G_{x}\right|_{p^{\prime}}^{2}$ it follows that the only possibilities are either:

$G_{x} \cap X=2^{6} A_{7}<\Omega_{7}(q)$ with $q$ either 3 or 5 , or $G_{x} \cap X=2^{n-1} A_{n}<\Omega_{n}(3)$ with $n=7,9$, or 11 .

In each case the fact that $k$ divides $2(v-1)$ forces $v>k^{2}$, a contradiction.

$\mathcal{C}_{3}$ ) If $G_{x} \in \mathcal{C}_{3}$ then $G_{x} \cap X=\Omega_{a}\left(q^{t}\right) . t$ with $n=a t$. Since $a$ and $t$ are odd, $a=2 r+1<\frac{n}{2}$, so

$$
\left|G_{x}\right|_{p^{\prime}}=t \prod_{i=1}^{r}\left(q^{2 i t}-1\right),
$$

and since $k$ divides $2\left(\left|G_{x}\right|_{p^{\prime}}, v-1\right)$, it is too small to satisfy $k^{2}>v$.

$\mathcal{C}_{4}$ ) If $G_{x} \in \mathcal{C}_{4}$ then it stabilises a tensor product of nonsingular subspaces, but these have to be of odd dimension and so $G_{x}$ is too small.

$\mathcal{C}_{5}$ ) If $G_{x} \in \mathcal{C}_{5}$ then $G_{x} \cap X=\Omega_{n}\left(q_{0}\right) . a$, with $q=q_{0}^{b}$ for some prime $b$, and $a \leq 2$ with $a=2$ if and only $b=2$. The inequality $|G|<\left|G_{x}\right|\left|G_{x}\right|_{p^{\prime}}^{2}$ forces $b=2$. If $n=2 m+1$ then $k$ divides $2\left|G_{x} \cap X\right|=q_{0}^{m^{2}}\left(q_{0}^{2 m}-1\right) \ldots\left(q_{0}^{2}-1\right)$, but $v=q^{m^{2}}\left(q_{0}^{2 m}+\right.$ $1) \ldots\left(q_{0}^{2}+1\right)$, so $k$ is prime to $q$ and therefore $\left(v-1,\left(q^{2 m}-1\right) \ldots\left(q_{0}^{2}-1\right)\right)$ is too small.

$\left.\mathcal{C}_{6}\right), \mathcal{C}_{7}$ ), and $\mathcal{C}_{8}$ ) In the cases $\mathcal{C}_{6}$ and $\mathcal{C}_{8}$, the classes are empty, and for $\mathcal{C}_{7}$ we see $G_{X} \cap X$ stabilises the tensor product power of a non-singular space, but it is too small to satisfy $|G|<\left|G_{x}\right|^{3}$.

$\mathcal{S})$ Now consider the case in which $G_{x}$ is a simple group not contained in any of the Aschbacher collection of subgroups of $G$. As in the symplectic section, we only need to consider the following possibilities:

(1) $G_{X} \cap X=G_{2}(q)<\Omega_{7}(q)=X$ with $q$ odd,

(2) $G_{x} \cap X=S p_{6}(2)<\Omega_{7}(p)$ with $p$ either 3 or 5 , or

(3) $G_{x} \cap X=S_{9}<\Omega_{7}(3)$.

In all three cases as $k$ divides $2\left(v-1,\left|G_{x}\right|\right)$ it is too small.

This completes the proof of Lemma 14.

\section{$6 X$ is an orthogonal group of even dimension}

In this section $X=P \Omega_{2 m}^{\epsilon}(q)$, with $m \geq 4$. We write $\beta_{+}=\left\{e_{1}, f_{1}, \ldots, e_{m}, f_{m}\right\}$ for a standard basis for $V$ in the $O_{2 m}^{+}$-case, and $\beta_{-}=\left\{e_{1}, f_{1}, \ldots, e_{m-1}, f_{m-1}, d, d^{\prime}\right\}$ in the $O_{2 m}^{-}$-case.

Lemma 15 The group $X$ is not $P \Omega_{2 m}^{\epsilon}(q)$, with $m \geq 4$. 
Proof As before, we take $G_{x}$ to be in one of the Aschbacher families of subgroups of $G$, or a simple group not contained in any of these families, and analyse each case separately. We postpone until the end of the proof the case where $(m, \epsilon)=(4,+)$ and $G$ contains a triality automorphism.

$\mathcal{C}_{1}$ ) If $G_{x} \in \mathcal{C}_{1}$ then we have two possibilities.

First assume $G_{x}$ stabilises a totally singular $i$-space, and suppose that $i<m$. If $i=m-1$ and $\epsilon=+$, then $G_{x}=P_{m, m-1}$, otherwise $G_{x}=P_{i}$. In any case there is a unique subdegree of $X$ that is a power of $p$ (except in the case where $\epsilon=+, m$ is odd, and $G_{x}=P_{m}$ or $P_{m-1}$ ). On the other hand, the highest power of $p$ dividing $v-1$ divides $q^{2}$ or 8 , so $k$ is too small.

Now consider $G_{x}=P_{m}$ in the case $X=P \Omega_{2 m}^{+}(q)$, and note that in this case $P_{m-1}$ and $P_{m}$ are the stabilisers of totally singular $m$-spaces from the two different $X$-orbits. If $m$ is even then

$$
x=\left\langle e_{1}, \ldots, e_{m}\right\rangle, y=\left\langle f_{1}, \ldots, f_{m}\right\rangle
$$

are in the same $X$-orbit, and the size of the $G_{x}$-orbit of $y$ is a power of $p$. However the highest power of $p$ dividing $v-1$ is $q$, so $k$ is too small.

If $m$ is odd, $m \geq 5$, then $v=\left(q^{m-1}+1\right)\left(q^{m-2}+1\right) \ldots(q+1)>q^{\frac{m(m-1)}{2}}$. Let

$$
x=\left\langle e_{1}, \ldots, e_{m}\right\rangle, y=\left\langle e_{1}, f_{2}, \ldots, f_{m}\right\rangle .
$$

Then $x$ and $y$ are in the same $X$-orbit, and the index of $G_{x y}$ in $G_{x}$ has $p^{\prime}$-part dividing $q^{m}-1$. The highest power of $p$ dividing $v-1$ is $q$ so $k$ divides $2 q\left(q^{m}-1\right)$, and the inequality $v<k^{2}$ implies $m=5$. In this case the action is of rank three, with nontrivial subdegrees

$$
\frac{q\left(q^{2}+1\right)\left(q^{5}-1\right)}{q-1} \text { and } \frac{q^{6}\left(q^{5}-1\right)}{q-1}
$$

Therefore $k$ divides

$$
\frac{2 q\left(q^{5}-1\right)}{q-1},
$$

and $v<k^{2}$ implies $k$ is either $2 q\left(q^{4}+q^{3}+q^{2}+q+1\right)$ or $q\left(q^{4}+q^{3}+q^{2}+q+1\right)$, but neither of these satisfies the equality $k(k-1)=2(v-1)$.

Now suppose $G_{x}=N_{i}$. First let $i=1$. The subdegrees of $X$ are (see [5]):

$$
\begin{aligned}
& q^{2 m-2}-1, \frac{q^{m-1}\left(q^{m-1}+\epsilon\right)}{2}, \frac{q^{m-1}\left(q^{m-1}-\epsilon\right)(q-1)}{4}, \text { and } \frac{q^{m-1}\left(q^{m-1}+\epsilon\right)(q-3)}{4} \text { if } q \equiv 1 \bmod 4, \\
& q^{2 m-2}-1, \frac{q^{m-1}\left(q^{m-1}-\epsilon\right)}{2}, \frac{q^{m-1}\left(q^{m-1}-\epsilon\right)(q-3)}{4}, \text { and } \frac{q^{m-1}\left(q^{m-1}+\epsilon\right)(q-3)}{4} \text { if } q \equiv 3 \bmod 4, \\
& \text { and } \\
& q^{2 m-2}-1, \frac{q^{m}\left(q^{m-1}-\epsilon\right)}{2}, \text { and } \frac{q^{m-1}\left(q^{m-1}+\epsilon\right)(q-2)}{2} \text { if } q \text { is even. }
\end{aligned}
$$

Here $k$ divides twice the highest common factor of the subdegrees, and in every case this is too small for $k$ to satisfy $v<k^{2}$.

Now let $G_{x}=N_{i}^{\epsilon_{1}}$, with $1<i \leq m$, and $\epsilon_{1}= \pm$ present only if $i$ is even. If $q$ is odd, as in the odd-dimensional case $S O_{i-1}(q) \times S O_{n-i-1}(q) \leq G_{x} \cap G_{x}^{g}$ for some 
$g \in G \backslash G_{x}$. Since $k$ and $p$ are coprime $k<8 q^{m} \log _{p} q$, contrary to $v<k^{2}$. Now assume $q$ is even. Then $i$ is also even.

If $i=2$ then we can find $g_{1}, g_{2} \in G \backslash G_{X} \cap X$ such that $\left(G_{x} \cap X\right) \cap\left(G_{x} \cap\right.$ $X)^{g_{1}} \geq S O_{n-4}^{+}(q)$ and $\left(G_{x} \cap X\right) \cap\left(G_{x} \cap X\right)^{g_{2}} \geq S O_{n-4}^{-}(q)$. Therefore $k$ divides $2\left(q-\epsilon_{1}\right)\left(q^{m-1}-\epsilon \epsilon_{1}\right)\left(\log _{2} q\right)_{2^{\prime}}$, so $k^{2}<v$.

If $2<i \leq m$ then we can find $g \in G \backslash G_{X} \cap X$ such that $\left(G_{x} \cap X\right) \cap\left(G_{x} \cap X\right)^{g} \geq$ $S O_{i-2}^{\epsilon_{1}}(q) \times S O_{n-i-2}^{\epsilon_{2}}(q)$, with $\epsilon_{2}=\epsilon \epsilon_{1}$. It follows that $k$ divides

$$
\left(q^{\frac{i}{2}}-\epsilon_{1}\right)\left(q^{\frac{i-2}{2}}+\epsilon_{1}\right)\left(q^{\frac{n-i}{2}}+\epsilon_{2}\right)\left(q^{\frac{n-i-2}{2}}+\epsilon_{2}\right)\left(\log _{2} q\right)_{2^{\prime}},
$$

forcing $k^{2}<v$, a contradiction.

$\mathcal{C}_{2}$ ) If $G_{x} \in \mathcal{C}_{2}$ then $G_{x}$ stabilises a decomposition $V=V_{1} \oplus \cdots \oplus V_{a}$ of subspaces of equal dimension, say $b$, so $n=a b$. Here we have three possibilities.

First assume all the $V_{i}$ are nonsingular and isometric. (Also, if $b$ is odd then so is $q$ ). If $b=1$ then the inequality $|G|<2\left|G_{x}\right|\left|G_{x}\right|_{p^{\prime}}^{2}$ implies $G_{x} \cap X=2^{n-2} A_{n}$, with $n$ being either 8 or 10 and $X$ either $P \Omega_{8}^{+}(3)$ or $P \Omega_{10}^{-}$(3) respectively. (Note that if $X=P \Omega_{8}^{+}(5)$ then the maximality of $G_{x}$ in $G$ forces $G \leq X .2$ ([16]), so $G_{x}$ is too small). In the first case, $k$ divides 112 , and in the second it is a power of 2 . Both contradict the inequality $v<k^{2}$.

Now let $b=2$. If $q>2$ then we can find $g \in G \backslash G_{x}$ so that $G_{x} \cap G_{x}^{g}$ contains the stabiliser of $V_{3} \oplus \cdots \oplus V_{a}$. From this it follows that $k \leq 2 a(a-1)$. $(2(q+1))^{2} \mid$ Out $X \mid$, and from $v<k^{2}$ we obtain $n=8$ and $q=3$. If $q=2$ then we can find $g \in G \backslash G_{x}$ so that $G_{x} \cap G_{x}^{g}$ contains the stabiliser of $V_{4} \oplus \cdots \oplus V_{a}$, and in this case $k$ is at most $2 a(a-1)(a-2)(2(q+1))^{3} \mid$ Out $X \mid$, and so $n=8$ or 10 . Using the condition that $k$ divides $2(v-1)$ we rule out these three cases.

Finally let $b>2$. The inequality $|G|<2\left|G_{x}\right|\left|G_{x}\right|_{p^{\prime}}^{2}$ forces $b=m$, (and so $\epsilon=+$ ). Let $\delta$ be the type of the $V_{i}$ if $m$ is even. Assume first that $m=4$. Then

$$
v=\frac{q^{8}\left(q^{2}+1\right)^{2}\left(q^{4}+q^{2}+1\right)}{4}
$$

if $\delta=+$, and

$$
v=\frac{q^{8}\left(q^{6}-1\right)\left(q^{2}-1\right)}{4}
$$

if $\delta=-$. In the first case, $\left(q^{2}-1, v-1\right) \leq 2$ and 4 does not divide $v-1$, so $k$ divides $6\left(\log _{p} q\right)_{2^{\prime}}$, contrary to $v<k^{2}$. In the latter case, $v$ is even and divisible by $\left(q^{2}-1\right)$, and $k$ divides the odd part of $3\left(q^{2}+1\right)^{2} \log _{p} q$, again contrary to $v<k^{2}$. Hence $m \geq 5$, and we argue as in $\mathcal{C}_{1}$.

In the case where $m$ and $q$ are odd, $a=2$, and $V_{1}, V_{2}$ are similar but not isometric, we also argue as in $\mathcal{C}_{1}$.

Now consider the case $\epsilon=+, a=2$, and $V_{1}$ and $V_{2}$ totally singular. If $m=4$, then we can apply a triality automorphism of $X$ to get to the case $G_{x}=N_{2}^{+}$, which we have ruled out in $\mathcal{C}_{1}$. Assume then that $m \geq 5$. Then

$$
v=\frac{q^{\frac{m(m-1)}{2}}\left(q^{m-1}+1\right)\left(q^{m-2}+1\right) \ldots(q+1)}{2^{e}},
$$


where $e$ is either 0 or $1([17,4.2 .7])$, so

$$
v>\frac{q^{m(m-1)}}{2} .
$$

However, there exists $g \in G \backslash G_{x}$ such that $G L_{m-2}(q) \leq G_{x} \cap G_{x}^{g}$, and so $k$ divides $2\left(q^{m}-1\right)\left(q^{m-1}-1\right) \log _{p} q$, and in fact $(k, v) \leq 2$ implies $k$ divides twice the odd part of $\frac{\left(q^{m}-1\right)\left(q^{m-1}-1\right) \log _{p} q}{q+1}$, which is contrary to $k^{2}<v$.

$\mathcal{C}_{3}$ ) If $G_{x} \in \mathcal{C}_{3}$, then $G_{x}$ is an extension field subgroup, and there are two possibilities ([17]).

First assume $G_{x}=N_{G}\left(\Omega_{\frac{n}{s}}^{\delta}\left(q^{s}\right)\right.$ ), with $s$ a prime and $\delta= \pm$ if $\frac{n}{s}$ is even (and empty otherwise). The inequality $|G|<\left|G_{x}\right|^{3}$ forces $s=2$. If $q$ is odd, then by Lemma 9 we see that a parabolic degree of $G_{x}$ divides $k$, and so it follows that $k$ is even, but since $v$ is even then 4 does not divide $k$, which is a contradiction.

If $q$ is even then $m$ is also even, and

$$
v=\frac{q^{\frac{m^{2}}{2}}\left(q^{2 m-2}-1\right)\left(q^{2 m-2}-1\right) \ldots\left(q^{2}-1\right)}{2^{e}},
$$

with $e \leq 2([17,4.3 .14,4.3 .16])$. As $k$ divides $2(v-1)$ it is prime to $q^{2}-1$, and it follows that $k^{2}<v$, another contradiction.

Now let $G_{x}=N_{G}\left({ }^{\wedge} G U_{m}(q)\right)$, with $\epsilon=(-1)^{m}$. If $q$ is odd, then as in the symplectic case $q+1$ divides $v$ and $k$, which is a contradiction.

So let $q$ be even. If $m=4$ then applying a triality automorphism of $X$ the action of $G$ becomes that of $N_{2}^{-}$, which has been ruled out in the case $\mathcal{C}_{1}$. So let $m \geq 5$. Now, $G_{x}$ is the stabiliser of a hermitian form [, ] on $V$ over $G F\left(q^{2}\right)$ such that the quadratic form $Q$ preserved by $X$ satisfies $Q(v)=[v, v]$ for $v \in V$. Let $W$ be a nonsingular 2-dimensional hermitian subspace over $G F\left(q^{2}\right)$. Then $W$ over $G F(q)$ is of type $O_{4}^{+}$. The pointwise stabiliser of $W^{\perp}$ in $G_{x} \cap X$ is $G U_{2}(q)$, which is properly contained in the pointwise stabiliser of $W^{\perp}$ in $X$. Thus we can find $g \in G \backslash G_{x}$ so that $G U_{m-2}(q) \leq G_{x} \cap G_{x}^{g}$. Then $k$ divides $2\left(q^{m}-(-1)^{m}\right)\left(q^{m-1}-(-1)^{m-1}\right) \log _{p} q$, contrary to $v<k^{2}$.

$\mathcal{C}_{4}$ ) If $G_{x} \in \mathcal{C}_{4}$ then $G_{x}$ stabilises an asymmetric tensor product, so either $G_{x}=N_{G}\left(P S p_{a}(q) \times P S p_{b}(q)\right)$ with $a$ and $b$ distinct even numbers, or $G_{x}=$ $N_{G}\left(P \Omega_{a}^{\epsilon_{1}}(q) \times P \Omega_{b}^{\epsilon_{2}}(q)\right)$ with $a, b \geq 3$ and $n=a b$. The inequality $|G|<$ $2\left|G_{x}\right|\left|G_{x}\right|_{p^{\prime}}^{2}$ implies $n=8$ and $G_{x}=N_{G}\left(P S p_{2}(q) \times P S p_{4}(q)\right)$. Applying a triality automorphism of $X$, the action becomes that of $N_{3}$, a case that has been ruled out in $\mathcal{C}_{1}$.

$\mathcal{C}_{5}$ ) If $G_{x} \in \mathcal{C}_{5}$ then it is a subfield subgroup. The inequality $|G|<2\left|G_{x}\right|\left|G_{x}\right|_{p^{\prime}}^{2}$ implies $G_{x} \cap X=P \Omega_{2 m}^{\delta}\left(q_{0}\right) \cdot 2^{e}<P \Omega_{2 m}^{+}(q)=X$, with $q=q_{0}^{2}$ and $e \leq 2([17,4.5 .10])$, so

$$
v>\frac{q_{0}^{2 m^{2}-m}}{4} .
$$

Now, $G_{x}$ stabilises a $G F\left(q_{0}\right)$-subspace $V_{0}$ of $V$. Let $U_{0}$ be a 2-subspace of $V_{0}$ of type $O_{2}^{+}\left(q_{0}\right)$, and $U$ a subspace of $V$ of type $O_{2}^{+}(q)$ containing $U_{0}$. There exists 
an element $g \in G \backslash G_{x}$ that stabilises $U^{\perp}$ pointwise, from this it follows that $G_{x} \cap$ $G_{x}^{g}$ involves $P \Omega_{2 m-2}^{\delta}\left(q_{0}\right)$. This implies that $k$ divides $2\left(q_{0}^{m}-\delta\right)\left(q_{0}^{m-1}+\delta\right) \mid$ Out $X \mid$, which contradicts the inequality $v<k^{2}$.

$\mathcal{C}_{6}$ ) If $G_{x} \in \mathcal{C}_{6}$, it is an extraspecial normaliser. From $|G|<\left|G_{x}\right|^{3}$ we have $G_{x} \cap$ $X=2^{6} A_{8}<P \Omega_{8}^{+}(3)=X$. Applying a triality automorphism of $X$, we have one of the cases already ruled out in $\mathcal{C}_{2}$.

$\mathcal{C}_{7}$ ) If $G_{x} \in \mathcal{C}_{7}$, then it stabilises a symmetric tensor product of $a$ spaces of dimension $b$, with $n=b^{a}$. Here $G_{x}$ is too small.

$\mathcal{C}_{8}$ ) In this case this class is empty.

$\mathcal{S}$ ) Now consider the case in which $G_{x}$ is an almost simple group (modulo scalars) not contained in any of the Aschbacher subgroups of $G$. For $n \leq 10$, the subgroups $G_{x}$ are listed in [15] and [16]. Since $|G|<2\left|G_{x}\right|\left|G_{x}\right|_{p^{\prime}}^{2}$, we have one of the following:

(1) $\Omega_{7}(q)<P \Omega_{8}^{+}(q)$,

(2) $\Omega_{8}^{+}(q)<P \Omega_{8}^{+}(q)$ with $q=3,5$, or 7 , or

(3) $A_{9}<\Omega_{8}^{+}(q), A_{12}<\Omega_{10}^{-}(2), A_{12}<P \Omega_{10}^{+}(3)$.

In the first case applying a triality automorphism gives an action on $N_{1}$, which was excluded in $\mathcal{C}_{1}$. In the second case the fact that $k$ divides $2\left(\left|G_{x}\right|, v-1\right)$ implies $k$ divides 20,6 , and $2 \cdot 3^{5} \cdot 5^{2}$, and so is too small. In the third case since 6 divides $v$, again $k$ is too small.

So $n \geq 12$. If $n>14$, then by [18, Theorem 4.2] we need only to consider the cases in which $G_{x}^{\prime}$ is alternating on the deleted permutation module, and in fact $A_{17}<$ $\Omega_{16}^{+}(2)$ is the only group which is big enough. Again, since $v$ is divisible by $2 \cdot 3 \cdot 17$ we conclude $k$ is too small. Now let $n=12$, respectively 14 . If $X$ is alternating, we only have to consider $A_{13}<\Omega_{12}^{-}(2)$, respectively $A_{16}<\Omega_{14}^{+}(2)$, however $k$ divides $2\left(v-1,\left|G_{x}\right|\right)$, so $k^{2}<v$, a contradiction. If $X$ is not alternating, then again since $\left|G_{x}\right|<q^{2 n+4}$ by [18, Theorem 4.2] it follows that $\left|G_{x}\right|<q^{28}$, respectively $\left|G_{x}\right|<$ $q^{32}$. On the other hand, from $|G|<2\left|G_{x}\right|\left|G_{x}\right|_{p^{\prime}}^{2}$ we obtain $\left|G_{x}\right|_{p^{\prime}}>\frac{q^{19}}{\sqrt{2}}$, respectively $\left|G_{x}\right|_{p^{\prime}}>q^{29}$. We can now see (cf. [19, Sections 2, 3, and 5]) that no sporadic or Lie type group will do for $G_{x}$.

Finally assume that $X=P \Omega_{8}^{+}(q)$, and $G$ contains a triality automorphism. The maximal groups are determined in [16]. If $G_{x} \cap X$ is a parabolic subgroup of $X$, then it is either $P_{2}$ or $P_{134}$. The first was ruled out in $\mathcal{C}_{1}$, so consider the latter. In this case

$$
v=\frac{\left(q^{6}-1\right)\left(q^{4}-1\right)}{(q-1)^{3}}>q^{11},
$$

and $(3, q) q$ is the highest power of $p$ dividing $v-1$. Since $X$ has a unique suborbit of size a power of $p$ (by Lemma 10), we have $k<2 q(3, q)$, which contradicts $v<k^{2}$.

Now, by [16] and $|G|<\left|G_{x}\right|\left|G_{x}\right|_{p^{\prime}}^{2}$, the only cases we have to consider are $G_{2}(q)$ for any $q$ and $\left(2^{9}\right) L_{3}(2)$ for $q=3$. In the first case,

$$
v=\frac{q^{6}\left(q^{4}-1\right)^{2}}{(q-1,2)^{2}},
$$


and Lemma 9 applied to $G_{2}(q)$ implies $G_{x B}$ is contained a parabolic subgroup, so $\frac{q^{6}-1}{q-1}$ divides $k$. However $k$ is prime to $q+1$, which is a contradiction. In the second case, $k$ divides 28 , which is too small.

This completes the proof of Lemma 15.

\section{$7 X$ is a unitary group}

Here $X=U_{n}(q)$ with $n \geq 3$, and $(n, q) \neq(3,2),(4,2)$, since these are isomorphic to $3^{2} \cdot Q_{8}$ and $P S p_{4}(3)$ respectively. We write $\beta=\left\{u_{1}, \ldots, u_{n}\right\}$ for an orthonormal basis of $V$.

Lemma 16 The group $X$ is not $U_{n}(q)$, with $n \geq 3$ and $(n, q) \neq(3,2),(4,2)$.

Proof As we have done throughout, we will consider $G_{x}$ to be in one of the Aschbacher families of subgroups of $G$, or a nonabelian simple group not contained in any of these families, and analyse each of these cases separately.

$\mathcal{C}_{1}$ ) If $G_{x}$ is reducible, then it is either a parabolic subgroup $P_{i}$, or the stabiliser $N_{i}$ of a nonsingular subspace.

First assume $G_{x}=P_{i}$ for some $i \leq \frac{n}{2}$. Then

$$
v=\frac{\left(q^{n}-(-1)^{n}\right)\left(q^{n-1}-(-1)^{n-1}\right) \ldots\left(q^{n-2 i+1}-(-1)^{n-2 i+1}\right)}{\left(q^{2 i}-1\right)\left(q^{2 i-2}-1\right) \ldots\left(q^{2}-1\right)} .
$$

There is a unique subdegree which is a power of $p$. The highest power of $p$ dividing $v-1$ is $q^{2}$, unless $n$ is even and $i=\frac{n}{2}$, in which case it is $q$, or $n$ is odd and $i=\frac{n-1}{2}$, in which case it is $q^{3}$. If $n=3$ then the action is 2-transitive, so consider $n>3$. Then $v>q^{i(2 n-3 i)}$, and so $v<k^{2}$, which is a contradiction.

Now suppose that $G_{x}=N_{i}$, with $i<\frac{n}{2}$, and take $x=\left\langle u_{1}, \ldots, u_{i}\right\rangle$. If we consider $y=\left\langle u_{1}, \ldots, u_{i-1}, u_{i+1}\right\rangle$, then $k$ divides $2\left(q^{i}-(-1)^{i}\right)\left(q^{n-i}-(-1)^{n-i}\right)$. However in this case

$$
v=\frac{q^{i(n-1)}\left(q^{n}-(-1)^{n}\right) \ldots\left(q^{n-i+1}-(-1)^{n-i+1}\right)}{\left(q^{i}-(-1)^{i}\right) \ldots(q+1)},
$$

and $v<k^{2}$ implies $i=1$. Therefore $k$ divides $2(q+1)\left(q^{n-1}-(-1)^{n-1}\right)$. Applying Lemma 9 to $U_{n-1}(q)$, we see $k$ is divisible by the degree of a parabolic action of $U_{n-1}(q)$. We check the subdegrees, and by the fact that $k$ divides $\left|G_{x}\right|^{2}$ as well as $k^{2}>v$ we conclude $n \leq 5$.

If $n=5$ then $k$ divides $2(q+1)\left(q^{4}-1\right)$ and is divisible by $q^{3}+1$, which can only happen if $q=2$, but in this case none of the possibilities for $k$ satisfy the equality $2(v-1)=k(k-1)$.

If $n=4$ then $q^{3}+1$ divides $k$, but $\left(2(v-1), q^{3}+1\right) \leq 2\left(q^{2}-q+1\right)$, which is a contradiction. 
Finally, if $n=3$ then $q+1$ divides $k$, but $q+1$ is prime to $v-1$, which is another contradiction.

$\mathcal{C}_{2}$ ) If $G_{x} \in \mathcal{C}_{2}$, then it preserves a partition $V=V_{1} \oplus \cdots \oplus V_{a}$ of subspaces of the same dimension, say $b$, so $n=a b$ and either the $v_{i}$ are nonsingular and the partition is orthogonal, or $a=2$ and the $V_{i}$ are totally singular.

First assume that the $V_{i}$ are nonsingular. If $b>1$, then taking

$$
x=\left\{\left\langle u_{1}, \ldots, u_{b}\right\rangle,\left\langle u_{b+1}, \ldots, u_{2 b}\right\rangle, \ldots\right\}
$$

and

$$
y=\left\{\left\langle u_{1}, \ldots, u_{b-1}, u_{b+1}\right\rangle,\left\langle u_{b}, u_{b+2}, \ldots, u_{2 b}\right\rangle, \ldots\right\},
$$

we see $k$ divides $2 a(a-1)\left(q^{b}-(-1)^{b}\right)^{2}$. From the inequality $v<k^{2}$ we have $n=4$ and $b=2$. Therefore

$$
v=\frac{q^{4}\left(q^{4}-1\right)\left(q^{3}+1\right)}{2\left(q^{2}-1\right)(q+1)}
$$

and $k$ divides $4\left(q^{2}-1\right)^{2}$. However, $(v-1, q+1)=(2, q+1)$, so $k$ divides $16(q-1)^{2}$, which is contrary to $v<k^{2}$.

If $b=1$ then $G_{x} \cap X=\hat{(}(q+1)^{n-1} S_{n}$. First let $n=3$, with $q>2$. Then

$$
v=\frac{q^{3}\left(q^{3}+1\right)\left(q^{2}-1\right)}{6(q+1)^{2}},
$$

and $k$ divides $12(q+1)^{2} \log _{p} q$. The inequality $v<k^{2}$ forces $q \leq 17$, but by the fact that $k$ divides $2(v-1)$ we rule out all these values. Now let $n>3$, and let $x=\left\{\left\langle u_{1}\right\rangle,\left\langle u_{2}\right\rangle, \ldots,\left\langle u_{n}\right\rangle\right\}$. If $q>3$ let $W=\left\langle u_{1}, u_{2}\right\rangle$. If we take $g \in G \backslash G_{x}$ acting trivially on $W^{\perp}$ we see $k$ divides $n(n-1)(q+1)^{2}$, contrary to $v<k^{2}$. If $q \leq 3$ then let $W=\left\langle u_{1}, u_{2}, u_{3}\right\rangle$. Taking $g \in G \backslash G_{x}$ acting trivially on $W^{\perp}$ we see that now $k$ divides $\frac{n(n-1)(n-2)(q+1)^{3}}{3}$, so $n \leq 6$ if $q=2$, or $n \leq 4$ if $q=2$. By the fact that $k$ divides $2(v-1)$ we rule these cases out.

Now assume that $a=2$ and both the $V_{i}$ 's are totally singular. Let $\left\{e_{1}, f_{1}, \ldots\right.$, $\left.e_{b}, f_{b}\right\}$ be a standard unitary basis. Take

$$
x=\left\{\left\langle e_{1}, \ldots, e_{b}\right\rangle,\left\langle f_{1}, \ldots, f_{b}\right\rangle\right\} \text {, and } y=\left\{\left\langle e_{1}, \ldots, e_{b-1}, f_{b}\right\rangle,\left\langle f_{1}, \ldots, f_{b-1}, e_{b}\right\rangle\right\} .
$$

Then $k$ divides $4\left(q^{n}-1\right)$. The inequality $v<k^{2}$ forces $n=4$, but then

$$
v=\frac{q^{4}\left(q^{3}+1\right)(q+1)}{2},
$$

so in fact $k$ divides $2\left(q^{2}+1\right)(q-1)$, contrary to $v<k^{2}$.

$\mathcal{C}_{3}$ ) If $G_{x} \in \mathcal{C}_{3}$ then it is a field extension group for some field extension of $G F(q)$ of odd degree $b$. From the inequality $|G|<2\left|G_{x}\right|\left|G_{x}\right|_{p^{\prime}}^{2}$ we have $b=3$ and $n=3$. Then

$$
v=\frac{q^{3}\left(q^{2}-1\right)(q+1)}{3}
$$


Therefore 4 does not divide $k$, and so $k<6 q^{2}\left(\log _{p} q\right)_{2^{\prime}}$. Since $v<k^{2}$, we have $q \leq 9$. With the condition that $k$ divides $2(v-1)$ we rule out these cases.

$\mathcal{C}_{4}$ ) If $G_{x} \in \mathcal{C}_{4}$ then it is the stabiliser of a tensor product of two nonsingular subspaces of dimensions $a>b>1$, but then the inequality $|G|<2\left|G_{x}\right|\left|G_{x}\right|_{p^{\prime}}^{2}$ is not satisfied.

$\mathcal{C}_{5}$ ) If $G_{x} \in \mathcal{C}_{5}$ then it is a subfield subgroup. We have three possibilities:

If $G_{x}$ is a unitary group of dimension $n$ over $G F\left(q_{0}\right)$, where $q=q_{0}^{b}$ with $b$ an odd prime, then $|G|<\left|G_{x}\right|^{3}$ implies $b=3$. However $|G|<2\left|G_{x}\right|\left|G_{x}\right|_{p^{\prime}}^{2}$ forces $q=8$ and $n \leq 4$, but in these cases since $k$ divides $2(v-1)$ it is too small.

If $G_{x} \cap X=P S O_{n}^{\epsilon}(q)$.2, with $n$ even and $q$ odd, then by Lemma $6 k$ is divisible by the degree of a parabolic action of $G_{x}$. Here $q+1$ divides $k$, and $\frac{q+1}{(4, q+1)}$ divides $v$. The fact that $k$ divides $2(v-1)$ forces $q=3$, so $v=2835$, but then $8 v-7$ is not a square, which is a contradiction.

Finally, if $G_{x}=N\left(P S p_{n}(q)\right)$, with $n$ even, then by Lemma $9 G_{x B}$ is contained on some parabolic subgroup, so $k$ is divisible by the degree of some parabolic action of $G_{x}$, and so is divisible by $q+1$. However $v$ is divisible by $\frac{q+1}{(q+1,2)}$, contradicting the fact that $k$ divides $2(v-1)$

$\mathcal{C}_{6}$ ) If $G_{x} \in \mathcal{C}_{6}$, then it is an extraspecial normaliser, and since $|G|<\left|G_{x}\right|^{3}$, we only have to consider the cases $G_{x} \cap X=3^{2} Q_{8}, 2^{4} A_{6}$, or $2^{4} S_{6}$, and $X=U_{3}(5)$, $U_{4}(3)$, and $U_{4}(7)$ respectively. In all cases the fact that $k$ divides $2\left(\left|G_{x}\right|, v-1\right)$ forces $k^{2}<v$, a contradiction.

$\mathcal{C}_{7}$ ) If $G_{x} \in \mathcal{C}_{7}$, then it stabilises a tensor product decomposition of $V_{n}(q)$ into $t$ subspaces $V_{i}$ of dimension $m$ each, so $n=m^{t}$. Since $m \geq 3$ and $t \geq 3$, we see $\left|G_{x}\right|$ is too small to satisfy $|G|<\left|G_{x}\right|^{3}$.

$\mathcal{C}_{8}$ ) This class is empty.

$\mathcal{S}$ ) Finally consider the case in which $G_{x}$ is an almost simple group (modulo the scalars) not contained in any of the Aschbacher families of subgroups. For $n \leq 10$ the subgroups $G_{x}$ are listed in [15, Chapter 5]. Since $|G|<\left|G_{x}\right|^{3}$, we only need to consider the following possibilities:

$L_{2}(7)$ in $U_{3}(3)$,

$A_{6} .2, L_{2}(7)$, and $A_{7}$ in $U_{3}(5)$,

$A_{6}$ in $U_{3}(11)$,

$L_{2}(7), A_{7}$, and $L_{2}(4)$ in $U_{4}(3)$,

$U_{4}(2)$ in $U_{4}(5)$,

$L_{2}(11)$ in $U_{5}(2)$, and

$U_{4}(3)$ and $M_{22}$ in $U_{6}(2)$.

Since $k$ divides $2\left(\left|G_{x}\right|, v-1\right)$, we have $k^{2}<v$ in all cases except in the case $L_{2}(7)<U_{3}(3)$. In this last case $v=36$, but then there is no $k$ such that $k(k-1)=$ $2(v-1)$, which is a contradiction.

If $n \geq 14$, then by [18] we have $|G|>\left|G_{x}\right|^{3}$, a contradiction. Hence $n=11,12$, or 13. By [18], $\left|G_{x}\right|$ is bounded above by $q^{4 n+8}$, and $|G|<2\left|G_{x}\right|\left|G_{x}\right|_{p^{\prime}}^{2}$ implies $\left|G_{x}\right|_{p^{\prime}}$ is bounded below by $q^{33}, q^{43}$, or $q^{53}$ respectively. Using the methods in $[18,19]$ we rule out all the almost simple groups $G_{x}$.

This completes the proof of Lemma 16, and hence if $X$ is a simple classical group, then it is either $P S L_{2}(7)$ or $P S L_{2}(11)$. 
Acknowledgements The results in the present paper were obtained with a grant from the Dirección General de Asuntos del Personal Académico, UNAM, during the course of my Ph.D. under the supervision of Martin W. Liebeck. I am very grateful to Martin for his most helpful ideas and guidance. I would also like to thank Jan Saxl for allowing me to view his notes before [27] was published, and Sasha Ivanov for providing me with these notes. Finally I thank the referees for their observations and suggestions, which have greatly improved this article.

\section{References}

1. Aschbacher, M. (1971). On collineation groups of symmetric block designs. J. Comb. Theory, 11, 272-281.

2. Aschbacher, M. (1984). On the maximal subgroups of the finite classical groups. Invent. Math., 76, 469-514.

3. Assmus, E. F. Jr., Mezzaroba, J. A., \& Salwach, C. J. (1977). Planes and biplanes. In Proceedings of the 1976 Berlin Combinatorics Conference. Vancerredle.

4. Assmus, E. F. Jr., \& Salwach, C. J. (1979). The (16,6,2) designs. Int. J. Math. Math. Sci., 2(2), 261281.

5. Bannai, E., Hao, S., \& Song, S.-Y. (1990). Character tables of the association schemes of finite orthogonal groups on the non-isotropic points. J. Comb. Theory Ser. A, 54, 164-200.

6. Cameron, P. J. (1973). Biplanes. Math. Z., 131, 85-101.

7. Colbourn, C. J., \& Dinitz, J. H. (1996). The CRC handbook of combinatorial designs. Boca Raton: CRC.

8. Conway, J. H., Curtis, R. T., Norton, S. P., Parker, R. A., \& Wilson, R. A. (1985). Atlas of finite groups. London: Oxford University Press.

9. Davies, H. (1987). Flag-transitivity and primitivity. Discret. Math., 63, 91-93.

10. Denniston, R. H. F. (1980). On biplanes with 56 points. Ars. Comb., 9, 167-179.

11. Hall, M. Jr., Lane, R., \& Wales, D. (1970). Designs derived from permutation groups. J. Comb. Theory, $8,12-22$.

12. Huppert, B. (1967). Endliche Gruppen. Berlin: Springer.

13. Hussain, Q. M. (1945). On the totality of the solutions for the symmetrical incomplete block designs $\lambda=2, k=5$ or 6. Sankhya, 7, 204-208.

14. Kantor, W. (1985). Classification of 2-transitive symmetric designs. Graphs Comb., 1, 165-166.

15. Kleidman, P. B. (1987). The subgroup structure of some finite simple groups. $\mathrm{PhD}$ thesis, University of Cambridge.

16. Kleidman, P. B. (1987). The maximal subgroups of the finite 8-dimensional orthogonal groups $P \Omega_{8}^{+}(q)$ and of their automorphism groups. J. Algebra, 110, 172-242.

17. Kleidman, P. B., \& Liebeck, M. W. (1990). The subgroup structure of the finite classical groups. London math. soc. lecture note series, Vol. 129. Cambridge: Cambridge Univ. Press.

18. Liebeck, M. W. (1985). On the orders of maximal subgroups of the finite classical groups. Proc. Lond. Math. Soc., 50, 426-446.

19. Liebeck, M. W. (1987). The affine permutation groups of rank 3. Proc. Lond. Math. Soc., 54, 477516.

20. Liebeck, M. W., \& Saxl, J. (1987). On the orders of maximal subgroups of the finite exceptional groups of Lie type. Proc. Lond. Math. Soc., 55, 299-330.

21. Liebeck, M. W., Saxl, J., \& Seitz, G. M. (1987). On the overgroups of irreducible subgroups of the finite classical groups. Proc. Lond. Math. Soc., 55, 507-537.

22. Liebeck, M. W., Praeger, C. E., \& Saxl, J. (1988). On the O’Nan-Scott theorem for finite primitive permutation groups. J. Aust. Math. Soc. (Ser. A), 44, 389-396.

23. Liebeck, M. W., Praeger, C. E., \& Saxl, J. (1988). On the 2-closures of finite permutation groups. J. Lond. Math. Soc., 37, 241-264.

24. O'Reilly Regueiro, E. (2005). On primitivity and reduction for flag-transitive symmetric designs. J. Comb. Theory Ser. A, 109, 135-148.

25. O'Reilly Regueiro, E. (2005). Biplanes with flag-transitive automorphism groups of almost simple type, with alternating or sporadic socle. Eur. J. Comb., 26, 577-584.

26. Salwach, C. J., \& Mezzaroba, J. A. (1978). The four biplanes with $k=9$. J. Comb. Theory Ser. A, 24, $141-145$.

27. Saxl, J. (2002). On finite linear spaces with almost simple flag-transitive automorphism groups. J. Comb. Theory Ser. A, 100(2), 322-348.

28. Seitz, G. M. (1973). Flag-transitive subgroups of Chevalley groups. Ann. Math., 97(1), 27-56. 